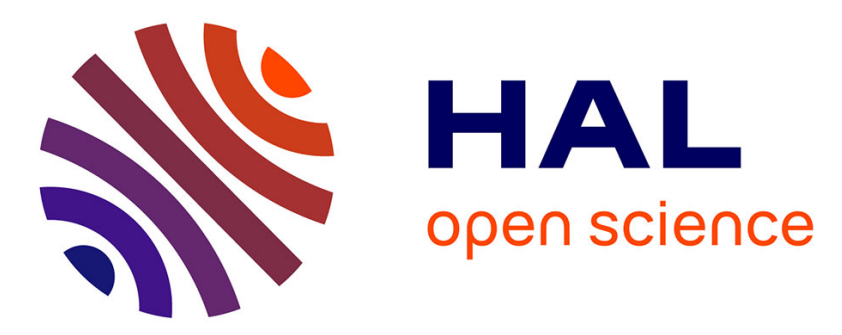

\title{
Numerical Study of Solutions of the 3D Generalized Kadomtsev-Petviashvili Equations for Long Times
}

\author{
Farid Hamidouche, Youcef Mammeri, Séraphin Mefire
}

\section{To cite this version:}

Farid Hamidouche, Youcef Mammeri, Séraphin Mefire. Numerical Study of Solutions of the 3D Generalized Kadomtsev-Petviashvili Equations for Long Times. Communications in Computational Physics, 2009, 6 (5), pp.1022 - 1062. 10.4208/cicp.2009.v6.p1022 . hal-01090387

\section{HAL Id: hal-01090387 \\ https://hal.science/hal-01090387}

Submitted on 3 Dec 2014

HAL is a multi-disciplinary open access archive for the deposit and dissemination of scientific research documents, whether they are published or not. The documents may come from teaching and research institutions in France or abroad, or from public or private research centers.
L'archive ouverte pluridisciplinaire HAL, est destinée au dépôt et à la diffusion de documents scientifiques de niveau recherche, publiés ou non, émanant des établissements d'enseignement et de recherche français ou étrangers, des laboratoires publics ou privés. 


\title{
Numerical study of solutions of the 3D generalized Kadomtsev-Petviashvili equations for long times
}

\author{
F. Hamidouche ${ }^{1}$, Y. Mammeri ${ }^{2}$ and S.M. Mefire ${ }^{3}$ \\ ${ }^{1}$ Laboratoire de Mathématiques, CNRS UMR 8628, Université Paris-Sud 11, \\ 91405 Orsay Cedex, France. \\ ${ }^{2}$ Laboratoire de Mathématiques - Paul Painlevé, CNRS UMR 8524, \\ Université des Sciences et Technologies de Lille, \\ 59655 Villeneuve d'Ascq Cedex, France. \\ Email: Youcef.Mammeri@math.univ-lille1.fr \\ ${ }^{3}$ Laboratoire Amiénois de Mathématique Fondamentale et Appliquée, CNRS UMR 6140, \\ Université de Picardie, 33 r. Saint-Leu, 80039 Amiens Cedex 1, France. \\ Email: Seraphin.Mefire@u-picardie.fr
}

December 2008

\section{Contents}

1 Introduction $\quad 2$

2 Numerical discretizations $\quad \mathbf{5}$

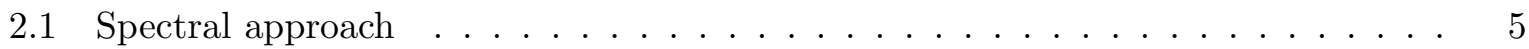

2.2 Time discretization . . . . . . . . . . . . . . . . 7

3 Long time behavior for localized initial data $\quad 10$

3.1 Non-generalized case: $p=1 \ldots \ldots \ldots \ldots \ldots$

3.1.1 Evolution in long time . . . . . . . . . . . . . . . . . 10

3.1 .2 With a datum of larger $L^{2}$-norm . . . . . . . . . . . . . . 13

3.2 Generalized case: $p>1 \ldots \ldots \ldots \ldots \ldots \ldots$

3.2 .1 The case $p=2 \ldots \ldots \ldots \ldots \ldots \ldots \ldots \ldots$

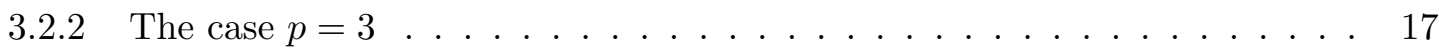

4 Transverse instabilities in the $y$ and $z$-directions $\quad 20$

4.1 Non-generalized case: $p=1 \ldots \ldots \ldots \ldots \ldots$

4.1 .1 Evolution in long time . . . . . . . . . . . . . . . . 21

4.1 .2 Periodicity in time of the surface . . . . . . . . . . . . 28

4.2 Generalized case: $p=2 \ldots \ldots \ldots \ldots$

4.2 .1 Evolution in long time . . . . . . . . . . . . . . 30 
4.2 .2 Periodicity in time of the surface . . . . . . . . . . 36

5 Transverse instability in the $z$-direction $\quad 38$

5.1 Evolution in long time . . . . . . . . . . . . . . . . . . 39

5.2 Periodicity in time of the crest $\ldots \ldots \ldots \ldots \ldots \ldots$

6 General conclusion $\quad 45$

Abstract. From a spectral method combined with a predictor-corrector scheme, we numerically study the behavior in time of solutions of the three-dimensional generalized Kadomtsev-Petviashvili equations. In a systematic way, the dispersion, the blow-up in finite time, the solitonic behavior and the transverse instabilities are numerically inspected.

Keywords. 3D-KP equations, spectral method, predictor-corrector method, dispersion, blow-up, soliton, transverse instability

MS Codes. 35B05, 35B35, 35Q53, 65L05, 65M70

\section{Introduction}

The propagation of long, dispersive and weakly nonlinear waves, essentially in the $x$-direction with weak transverse effects in the $y$-direction, is modelled by the Kadomtsev-Petviashvili (KP) equation [11],

$$
u_{t}+u_{x}+u u_{x}+u_{x x x}+a \partial_{x}^{-1} u_{y y}=0,
$$

called KP-I if $a=-1$ and KP-II if $a=1$, according to whether the surface tension is neglected or not. The approach in [11] for introducing (1) from the Korteweg de Vries (KdV) equation,

$$
u_{t}+u_{x}+u u_{x}+u_{x x x}=0
$$

can be extended to the context of two transverse variables. As explained by L. Molinet, J.-C. Saut \& N. Tzvetkov [15], in this context, if we aim at preserving the finite propagation speed properties of the transport operator $\partial_{t}+\partial_{x}$ for waves localized in the frequency regions $\frac{\left|\xi_{2}\right|}{\left|\xi_{1}\right|} \ll 1$ and $\frac{\left|\xi_{3}\right|}{\left|\xi_{1}\right|} \ll 1$, where $\xi_{1}, \xi_{2}$ and $\xi_{3}$ are the Fourier modes corresponding respectively to the space variables $x, y$ and $z$, we are led to consider the generalized operator

$$
\partial_{t}+\partial_{x}+\frac{1}{2}\left(\partial_{x}^{-1} \partial_{y y}+\partial_{x}^{-1} \partial_{z z}\right)
$$

where $\partial_{x}^{-1}$ denotes the anti-derivative, also defined such that $\widehat{\partial_{x}^{-1} u}\left(\xi_{1}, \xi_{2}, \xi_{3}\right):=\frac{\widehat{u}\left(\xi_{1}, \xi_{2}, \xi_{3}\right)}{\mathrm{i} \xi_{1}}$, and $\widehat{u}$ represents the Fourier transform of $u$. 
In this paper, we are concerned with the generalized Kadomtsev-Petviashvili equations in three-dimensional space:

$$
u_{t}+u^{p} u_{x}+u_{x x x}+a \partial_{x}^{-1} u_{y y}+b \partial_{x}^{-1} u_{z z}=0
$$

where $p \geq 1$, and the constants $a, b$ are normalized to \pm 1 . The separate term $u_{x}$ does not of course appear in (2) since a change of functions has now been applied $(u(x, y, z, t):=\widetilde{u}(x+$ $t, y, z, t))$. The mass and the energy

$\int_{\mathbb{R}^{3}} u^{2}(x, y, z, t) d x d y d z, \int_{\mathbb{R}^{3}}\left[\frac{u^{p+2}}{(p+1)(p+2)}-\frac{1}{2} u_{x}^{2}+\frac{a}{2}\left(\partial_{x}^{-1} u_{y}\right)^{2}+\frac{b}{2}\left(\partial_{x}^{-1} u_{z}\right)^{2}\right](x, y, z, t) d x d y d z$

are conserved by the flow associated with (2). Although the literature proposes an extensive list of (theoretical and numerical) works related with the KP equations in dimension two, namely with the Cauchy problem based on (1), see e.g. [3, 7, 8, 9, 10, 12, 18, 22, 25], we do not find the same range of references in the three-dimensional case. Some theoretical results concerning the behavior of solutions of (2) have been recently given in $[4,5,13,19]$. In particular, the existence as well as the non-existence of solitary waves are proved by A. De Bouard \& J.-C. Saut [4, 5], and J.-C. Saut establishes in [19] a result of blow-up in finite time for $a=b=-1$ and $p \geq 2$. In [13], Y. Liu continues this investigation and proves that, when a solution of (2), for $a=b=-1$ and $1 \leq p<4 / 3$, is initially close to an unstable solitary wave, then this solution blows up in finite time. Since no result has yet been proved for blow-up in finite time, with $a=b=1$ and $a b=-1$, there is considerable interest in performing numerical simulations aimed at studying the solutions of (2) in various situations.

The aim of this paper consists not only of inspecting numerically certain theoretical properties already stated, but above all of investigating, in diverse contexts, aspects not yet established, such as the blow-up in finite time, the dispersion, the solitonic behavior and the transverse instabilities.

This paper is subdivided into six sections. In Section 2, we discretize the Cauchy problem associated with (2), by combining a spectral method for the space discretization and a predictor-corrector scheme for the time discretization. Sections 3, 4 and 5 deal systematically with numerical simulations. Due to extensive numerical experiments performed throughout these sections, the paper is organized in such a way that each of these sections contains its own conclusions.

We study in Section 3 the propagation, based on (2), of localized initial data. The numerical observations show here that the effect of two negative transverse directions $(a=b=-1)$ is much less regularizing than the effect of two positive or "opposite" transverse directions. Indeed, in the context where $a=b=-1$, the discrete solution of the Cauchy problem blows up in finite 
time when for instance $p=2$, whereas the discrete solution in the context where $a=b=1$, or $a b=-1$, blows up in finite time when $p=3$.

In Section 4, our investigations concern the numerical study of the transverse instabilities in the $y$ and $z$-directions. Improperly, we talk about the solution stability instead of the orbital stability of the solitary wave close to this solution, namely a solitary wave for which the profile remains close to that solution (see e.g. [2]). By considering the line-soliton

$$
\Phi_{c}(x, t)=\left(\frac{(p+1)(p+2)}{2} c\right)^{1 / p} \operatorname{sech}^{2 / p}\left(\frac{p \sqrt{c}}{2}(x-c t)\right),
$$

for $p=1$, J.C. Alexander, R.L. Pego \& R.L. Sachs [1] characterize the unstable mode for (1), i.e. the solution of the linearized equation around $\Phi_{c}$, of the form $u(x, y, t)=\mathrm{e}^{\sigma t+\frac{2 \mathbf{i}_{\pi y}}{\lambda_{y}}} \widetilde{u}(x)$ with $\lambda_{y}>0$ a wavelength, $\operatorname{Re}(\sigma)>0$, and $\widetilde{u} \in L^{2}(\mathbb{R})$. They prove that KP-II does not admit unstable modes, and that KP-I admits unstable modes if and only if $\lambda_{y}>\frac{8 \pi}{c \sqrt{3}}$. A similar result of instability regarding KP-I is obtained in a nonlinear context by F. Rousset \& N. Tzvetkov [17]. In this section, we extend, to the 3D case, the approach considered by F. Hamidouche for studying numerically in [9] the transverse instabilities of solutions of $2 \mathrm{D}-\mathrm{KP}$ equations. We observe a stronger form of instability in the sense that solutions of (2), initially close to solitary waves, blow up in finite time - even for $p=1$. This study of instabilities is done here in the transverse directions, by associating with (2) the initial datum:

$$
u_{0}(x, y, z):=\Phi_{c(y, z)}(x, 0)
$$

where $c(y, z):=c\left(1+\varepsilon \cos \left(\frac{2 \pi y}{\lambda_{y}}+\frac{2 \pi z}{\lambda_{z}}\right)\right)$ is a perturbation of the velocity of the line-soliton, in an infinitesimal way in the $y$ and $z$-directions, $\lambda_{y}, \lambda_{z}>0$, and $\varepsilon>0$ is a fixed small value. When $p=1,2$ and $a=b=-1$, respectively when $p=2$ and $a b=-1$, we observe numerically that there exist two critical transverse wavelengths $0<\lambda_{c}^{1} \ll \lambda_{c}^{2}$, depending only on $c$, such that the discrete solution of (2) with such an initial datum $u_{0}$ blows up in finite time (in particular, the line-soliton is unstable under the flow of (2)) if $\lambda_{c}^{1}<\lambda_{y}<\lambda_{c}^{2}$ or $\lambda_{c}^{1}<\lambda_{z}<\lambda_{c}^{2}$. The existence of the critical value $\lambda_{c}^{2}$ translates the fact that the formal limit of the perturbed line-soliton, when the wavelengths $\lambda_{y}$ and $\lambda_{z}$ tend to infinity, is the $\mathrm{KdV}$ one of velocity $c(1+\varepsilon)$ which is stable [2]. These observations were foreseeable from the ones concerning KP-I. Another interesting observation is made when $p=1$ and $a=1, b=-1$. We notice that the discrete solution of (2) with this initial datum blows up if $\lambda_{c}^{1}<\lambda_{z}<\lambda_{c}^{2}$ and if the wavelength in the positive transverse direction is either small $\left(\lambda_{y}<\lambda_{c}^{1}\right)$ or very large $\left(\lambda_{y}>\lambda_{c}^{2}\right)$. It results then, namely when (2) is formed from KP-II with the addition of a negative transverse direction, that the solution of (2) can blow up, although the solution corresponding to KP-II is stable. In a symmetrical way, the 
same effects are noticed in the context where $p=1$ and $a=-1, b=1$. When (2) is formed from KP-I with the addition of a positive transverse direction, it follows that the solution of (2) can no longer blow up.

In Section 5, we deal with the study of the transverse instability in the $z$-direction by associating now with (2) another type of initial datum:

$$
u_{0}(x, y, z):=\psi_{c(z)}(x, y, 0)
$$

where

$\psi_{c}(x, y, t)=12 \alpha^{2} \frac{1-\beta \cosh (\alpha x-\omega t) \cos (\delta y)}{(\cosh (\alpha x-\omega t)-\beta \cos (\delta y))^{2}}, \beta=\sqrt{\frac{\delta^{2}-3 \alpha^{4}}{\delta^{2}}}, 3 \alpha^{4}<\delta^{2}, \omega=\frac{\delta^{2}+\alpha^{4}}{\alpha}, c=\frac{\omega}{\alpha}$.

The function $\psi_{c}$ is known as being the profile of the Zaitsev [24] traveling waves (see also $[14,20])$. It presents a fork phenomenon corresponding to values of $\beta$, typically for $\beta=0$ and $\beta=1$ (see e.g. [9]). In the case where $\beta=0, \psi_{c}$ forks to a $1 \mathrm{D}$-soliton of KP-I (this is denoted by $\Phi_{c}$ with $\left.p=1\right)$. In the situation where $\beta$ tends to 1 , an expansion of $\psi_{c}$, for $\alpha$ and $\delta$ close to 0 , leads us to a 2D-soliton of KP-I so called the lump-soliton. Our considerations in this section affect small values to $\alpha$ and $\delta$. The velocity is here slightly perturbed in the $z$-direction; $c(z):=c\left(1+\varepsilon \cos \left(\frac{2 \pi z}{\lambda_{z}}\right)\right), \lambda_{z}>0$, and $\varepsilon>0$ is a fixed small value. Our numerical experiments are performed in the special context $p=1$, with $a=-1$. It follows that the wavelength $\lambda_{z}$ characterizes the instability by transverse perturbations. More precisely, when $b=-1$, there exist two critical transverse wavelengths $0<\lambda_{c}^{1} \ll \lambda_{c}^{2}$, depending only on $c$, such that the solution of (2) with $u_{0}$ as initial datum blows up (and is hence unstable) if $\lambda_{c}^{1}<\lambda_{z}<\lambda_{c}^{2}$.

Finally, the general conclusion in Section 6 summarizes our main results.

\section{Numerical discretizations}

By combining a spectral approach with a predictor-corrector scheme, we discretize here the Cauchy problem based on (2).

\subsection{Spectral approach}

Spectral methods are very suitable to the discretization of equations such as (2). In fact, the non-linearity and the anti-derivative appearing in (2) are easily treated with such methods, contrary to finite element methods or finite volume methods. Finite difference discretizations of (2) can be an alternative, but the spectral methods are again more suited when we are looking for solutions of (2) in periodised domains. 
Let us consider the Cauchy problem based on (2), where $u_{0}$ denotes the initial datum:

$$
u(x, y, z, 0):=u_{0}(x, y, z)
$$

As in [21, 23], where (1) is considered, we will be concerned with solutions of (2) such that $u, u_{x}$, $u_{y}, u_{z}, u_{x x}, u_{y y}, u_{z z}$ vanish at infinity and furthermore $\partial_{x}^{-1} u_{y}, \partial_{x}^{-1} u_{z} \in L^{2}\left(\mathbb{R}^{3}\right), \partial_{x}^{-2} u_{y y}, \partial_{x}^{-2} u_{z z} \in$ $L^{2}\left(\mathbb{R}^{3}\right)$. For $\left(\xi_{1}, \xi_{2}, \xi_{3}\right) \in \mathbb{R}^{3}$, and $t \geq 0$, we use the notation:

$$
\widehat{u}\left(\xi_{1}, \xi_{2}, \xi_{3}, t\right):=\int_{-\infty}^{\infty} \int_{-\infty}^{\infty} \int_{-\infty}^{\infty} u(x, y, z, t) \mathrm{e}^{-\mathrm{i}\left(\xi_{1} x+\xi_{2} y+\xi_{3} z\right)} d x d y d z .
$$

By applying this Fourier transform to the equations of the considered Cauchy problem, it follows that:

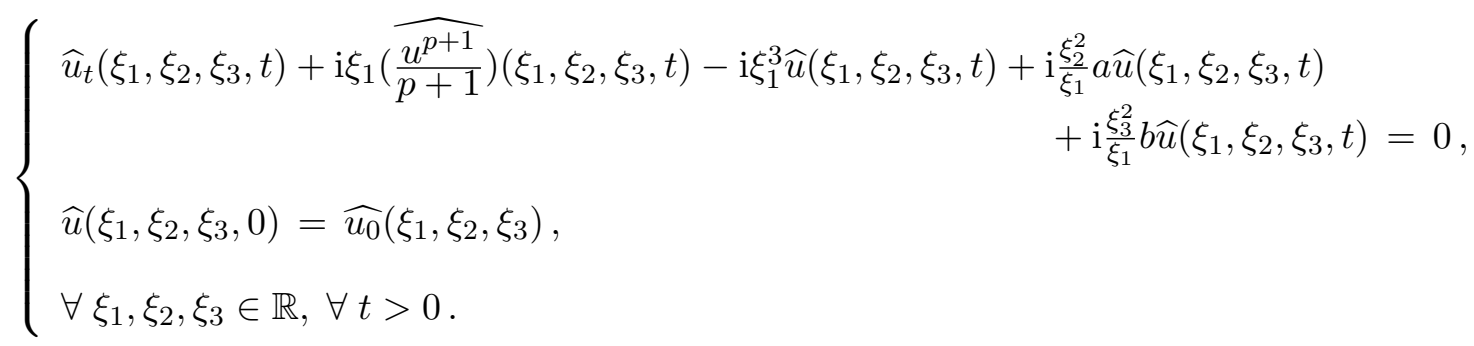

Looking moreover for solutions of (2) such that $u, u^{p+1}$ and $u_{t} \in L^{1}\left(\mathbb{R}^{3}\right) \cap L^{2}\left(\mathbb{R}^{3}\right)$, we notice from (3) in particular that: $\forall \xi_{2}, \xi_{3} \in \mathbb{R}, \forall t>0$,

$$
\lim _{\xi_{1} \longrightarrow 0} \widehat{u}\left(\xi_{1}, \xi_{2}, \xi_{3}, t\right)=0, \quad \lim _{\xi_{1} \longrightarrow 0} \frac{\widehat{u}\left(\xi_{1}, \xi_{2}, \xi_{3}, t\right)}{\xi_{1}}=0,
$$

provided that

$$
\widehat{u_{0}}\left(0, \xi_{2}, \xi_{3}\right)=0, \quad \lim _{\xi_{1} \longrightarrow 0} \frac{\widehat{u_{0}}\left(\xi_{1}, \xi_{2}, \xi_{3}\right)}{\xi_{1}}=0 .
$$

By giving in this way a sense to $\lim _{\xi_{1} \longrightarrow 0} \widehat{u}\left(\xi_{1}, \xi_{2}, \xi_{3}, t\right)$ and $\lim _{\xi_{1} \longrightarrow 0} \frac{\widehat{u}\left(\xi_{1}, \xi_{2}, \xi_{3}, t\right)}{\xi_{1}}$, the singularities for $\xi_{1}=0$ in (3) are overcome. Relations (4) enforce restrictions on the choice of $u_{0} \in L^{1}\left(\mathbb{R}^{3}\right) \cap$ $L^{2}\left(\mathbb{R}^{3}\right)$; we will be concerned with such an initial datum.

In view of numerical computations in a bounded and periodised domain $\Omega$, we now consider (2) in $[-K, K] \times[-L, L] \times[-M, M]=: \Omega$, where $K, L, M>0$ must be fixed values allowing us to express the relations (4) as below:

$$
\left\{\begin{aligned}
& \int_{-M}^{M} \int_{-L}^{L} \mathrm{e}^{-\mathrm{i}\left(\xi_{2} y+\xi_{3} z\right)}\left(\int_{-K}^{K} u_{0}(x, y, z) d x\right) d y d z=0 \\
& \int_{-M}^{M} \int_{-L}^{L} \mathrm{e}^{-\mathrm{i}\left(\xi_{2} y+\xi_{3} z\right)}\left(\int_{-K}^{K} \int_{-K}^{x} u_{0}\left(x_{1}, y, z\right) d x_{1} d x\right) d y d z=0 .
\end{aligned}\right.
$$


Let us consider now the discrete Fourier basis consisting of trigonometric polynomial functions: $\mathrm{e}^{\mathrm{i} k \frac{\pi}{K} x} \mathrm{e}^{\mathrm{i} l \frac{\pi}{L} y} \mathrm{e}^{\mathrm{i} m \frac{\pi}{M} z} ; \quad \mathrm{i}^{2}=-1, \quad-\frac{N_{x}}{2} \leq k \leq \frac{N_{x}}{2}-1, \quad-\frac{N_{y}}{2} \leq l \leq \frac{N_{y}}{2}-1, \quad-\frac{N_{z}}{2} \leq m \leq \frac{N_{z}}{2}-1$, where $N_{x}, N_{y}, N_{z} \in 2 \mathbb{N}^{\star}$ represent the numbers of modes. Let us set $N_{x}=\frac{2 K}{\Delta x}, N_{y}=\frac{2 L}{\Delta y}$ and $N_{z}=\frac{2 M}{\Delta z}$, where $\Delta x, \Delta y, \Delta z>0$. For any non periodic scalar function $f$, locally summable, we denote by $f_{K, L, M}$ the function equal to $f$ in $\Omega$ and extended outside of $\Omega$ as a periodic function of period equal to $2 K$ in the $x$-direction, $2 L$ in the $y$-direction, $2 M$ in the $z$-direction. The expression of $f_{K, L, M}$ in the considered discrete basis is as follows,

$$
f_{K, L, M}(x, y, z)=\sum_{k=-\frac{N_{x}}{2}, l=-\frac{N_{y}}{2}, m=-\frac{N_{z}}{2}}^{\frac{N_{x}}{2}-1, \frac{N_{y}}{2}-1, \frac{N_{z}}{2}-1} \widehat{f}\left(k \frac{\pi}{K}, l \frac{\pi}{L}, m \frac{\pi}{M}\right) \mathrm{e}^{\mathrm{i} k \frac{\pi}{K} x} \mathrm{e}^{\mathrm{i} l \frac{\pi}{L} y} \mathrm{e}^{\mathrm{i} m \frac{\pi}{M} z},
$$

with

$$
\widehat{f}\left(k \frac{\pi}{K}, l \frac{\pi}{L}, m \frac{\pi}{M}\right)=\frac{1}{(2 K)(2 L)(2 M)} \int_{-K}^{K} \int_{-L}^{L} \int_{-M}^{M} f(x, y, z) \mathrm{e}^{-\mathrm{i} k \frac{\pi}{K} x} \mathrm{e}^{-\mathrm{i} l \frac{\pi}{L} y} \mathrm{e}^{-\mathrm{i} m \frac{\pi}{M} z} d x d y d z .
$$

We are then concerned with a system of $N_{x} \times N_{y} \times N_{z}$ ordinary differential equations and $N_{x} \times N_{y} \times N_{z}$ unknowns, by reconsidering (3) and (4) with these notations, and hence projecting (3) in the Fourier basis. For each triplet $\left(\xi_{1}, \xi_{2}, \xi_{3}\right)$, find $\widehat{u}\left(\xi_{1}, \xi_{2}, \xi_{3}, t\right)$ satisfying

$$
\left\{\begin{array}{l}
\widehat{u}_{t}\left(\xi_{1}, \xi_{2}, \xi_{3}, t\right)+\mathrm{i} \xi_{1}\left(\widehat{\frac{u^{p+1}}{p+1}}\right)\left(\xi_{1}, \xi_{2}, \xi_{3}, t\right)+\left(-\mathrm{i} \xi_{1}^{3}+\mathrm{i} a \frac{\xi_{2}^{2}}{\xi_{1}}+\mathrm{i} b \frac{\xi_{3}^{2}}{\xi_{1}}\right) \widehat{u}\left(\xi_{1}, \xi_{2}, \xi_{3}, t\right)=0, \quad \forall t>0, \\
\widehat{u}\left(0, \xi_{2}, \xi_{3}, t\right)=\widehat{u_{0}}\left(0, \xi_{2}, \xi_{3}\right), \quad \forall t>0, \\
\widehat{u}\left(\xi_{1}, \xi_{2}, \xi_{3}, 0\right)=\widehat{u_{0}}\left(\xi_{1}, \xi_{2}, \xi_{3}\right),
\end{array}\right.
$$

where

$$
\left\{\begin{array}{l}
\xi_{1}=k \frac{\pi}{K}, \xi_{2}=l \frac{\pi}{L}, \xi_{3}=m \frac{\pi}{M}, \\
-\frac{N_{x}}{2} \leq k \leq \frac{N_{x}}{2}-1, \quad-\frac{N_{y}}{2} \leq l \leq \frac{N_{y}}{2}-1, \quad-\frac{N_{z}}{2} \leq m \leq \frac{N_{z}}{2}-1,
\end{array}\right.
$$

and the datum $u_{0}$ is subject to (5).

\subsection{Time discretization}

Many numerical methods can be applied for the time discretization of (6). In view of efficient numerical computations on long time intervals, we consider implicit methods such as AdamsMoulton methods (see e.g. [16]). Also, in view of flexible numerical implementations, we will restrict ourselves to one-step schemes. 
Let $\Delta t>0$ be the step of the time discretization, and set $t_{n}=n \Delta t$. For $n \in \mathbb{N}$, we denote by $\widehat{u}_{n}$ an approximation of $\widehat{u}\left(\xi_{1}, \xi_{2}, \xi_{3}, t_{n}\right)$ and by $u_{n}$ an approximation of $u\left(x, y, z, t_{n}\right)$, where $(x, y, z) \in \Omega$.

A possible way for numerically discretizing the partial differential equation in (6) consists of directly applying the Crank-Nicolson scheme, or a variant of this scheme as in the case of computations based on 2D-KP equations (see [9]). The following relations are then obtained: $\forall n \geq 0$,

$$
\widehat{u}_{n+1}=\widehat{u}_{n}+\frac{\Delta t}{2}\left(-\mathrm{i} \xi_{1}\left[\left(\frac{\widehat{u_{n+1}^{p+1}}}{p+1}\right)+\left(\widehat{\frac{u_{n}^{p+1}}{p+1}}\right)\right]+\mathrm{i}\left(\xi_{1}^{3}-\frac{\xi_{2}^{2}}{\xi_{1}} a-\frac{\xi_{3}^{2}}{\xi_{1}} b\right)\left[\widehat{u}_{n}+\widehat{u}_{n+1}\right]\right),
$$

with of course $\xi_{1} \neq 0, \xi_{2}$ and $\xi_{3}$ defined as in (7). A nonlinear system of $N_{x} \times N_{x} \times N_{z}$ equations and $N_{x} \times N_{x} \times N_{z}$ unknowns can thus be associated with (6) - (7) and solved with a fixed point iteration method, by considering from (8) a subsequence $\left(\widehat{u}_{n+1, r}\right)_{r}$ where we define, for each $n \geq 0, \widehat{u}_{n+1,0}:=\widehat{u}_{n}$, and also set $\widehat{u}_{n+1}:=\widehat{u}_{n+1, r+1}$ when the convergence of the subsequence is reached at the $(r+1)$ th iteration. However, this approach can be costly in CPU time, since such an initialization of fixed point iterations is not necessarily suitable for fast convergence (see e.g. $[16])$.

The method we consider for solving (6) - (7) consists of combining an Adams-Moulton scheme, for defining the fixed point iterations, with an Adams-Bashforth scheme for the initialization of these iterations. More precisely, restricting ourselves here to one-step schemes, we use the explicit Euler method for the predictor and the Crank-Nicolson method for the corrector. The resulting scheme is a predictor-corrector method and is of order two with respect to the time step (see e.g. [16]) under suitable regularities of the solution of (6) - (7).

By denoting by $m \geq 1$ a maximal number of iterations, the algorithm then considered for solving (6) - (7) is described as follows:

- Set $\widehat{u}_{0, m}:=\ldots:=\widehat{u}_{0,1}:=\widehat{u}_{0,0}:=\widehat{u_{0}}$.

- For $n=0,1, \ldots$, compute:

$$
\widehat{u}_{n+1,0}:=\widehat{u}_{n, m}+\Delta t\left(-\mathrm{i} \xi_{1}\left(\frac{\widehat{u_{n, m}^{p+1}}}{p+1}\right)+\mathrm{i}\left(\xi_{1}^{3}-\frac{\xi_{2}^{2}}{\xi_{1}} a-\frac{\xi_{3}^{2}}{\xi_{1}} b\right) \widehat{u}_{n, m}\right),
$$

- For $r=0,1, \ldots, m-1$, 


$$
\begin{array}{r}
\widehat{u}_{n+1, r+1}:=\widehat{u}_{n, m}+\frac{\Delta t}{2}\left(-\mathrm{i} \xi_{1}\left(\frac{\widehat{u_{n+1, r}^{p+1}}}{p+1}\right)+\mathrm{i}\left(\xi_{1}^{3}-\frac{\xi_{2}^{2}}{\xi_{1}} a-\frac{\xi_{3}^{2}}{\xi_{1}} b\right) \widehat{u}_{n+1, r}-\mathrm{i} \xi_{1}\left(\frac{\widehat{u_{n, m}^{p+1}}}{p+1}\right)\right. \\
\left.+\mathrm{i}\left(\xi_{1}^{3}-\frac{\xi_{2}^{2}}{\xi_{1}} a-\frac{\xi_{3}^{2}}{\xi_{1}} b\right) \widehat{u}_{n, m}\right) .
\end{array}
$$

The iterations are stopped in one of the two following cases:

- when $\frac{\left\|u_{n+1, r+1}-u_{n+1, r}\right\|_{l^{2}(\Omega)}}{\left\|u_{n+1,0}\right\|_{l^{2}(\Omega)}} \leq \tau$, with $\tau>0$ a fixed tolerance. We then set $u_{n+1}:=$ $u_{n+1, r+1}$;

- or when $r=m-1$. Here, we set $u_{n+1}:=u_{n+1, m}$. We mention that the step $\Delta t$ can be reduced in this case, in order to improve the previous relative error.

This algorithm provides the solution $\widehat{u}_{n}\left(\xi_{1}, \xi_{2}, \xi_{3}\right)$, where $\xi_{1} \neq 0, \xi_{2}, \xi_{3}$ are defined as in (7), and where we consider:

- For $n=1,2, \ldots$,

$$
\widehat{u}_{n}\left(0, \xi_{2}, \xi_{3}\right):=\widehat{u_{0}}\left(0, \xi_{2}, \xi_{3}\right) \text {. }
$$

Remark 2.1 A practical way to choose a suitable (initial) step $\Delta t$ in the previous algorithm is the one involving the parameters $\Delta x, \Delta y, \Delta z$ as follows:

$$
\Delta t<\frac{2 \Delta x}{\pi\left(\frac{\pi^{2}}{(\Delta x)^{2}}+\frac{\Delta x K}{(\Delta y)^{2}}+\frac{\Delta x K}{(\Delta z)^{2}}+\left\|u_{n}\right\|_{L^{\infty}(\Omega)}^{p}\right)} .
$$

Of course, since from (9),

$$
\widehat{u}_{n+1, r+1}-\widehat{u}_{n+1} \approx\left(\widehat{u}_{n+1, r}-\widehat{u}_{n+1}\right)\left(\mathrm{i} \frac{\Delta t}{2}\left(\xi_{1}^{3}-\frac{\xi_{2}^{2}}{\xi_{1}} a-\frac{\xi_{3}^{2}}{\xi_{1}} b\right)\right)-\mathrm{i} \xi_{1} \frac{\Delta t}{2}\left(\left(\frac{\widehat{u_{n+1, r}^{p+1}}}{p+1}\right)-\left(\frac{\widehat{u_{n+1}^{p+1}}}{p+1}\right)\right),
$$

it follows in the case where $p \in \mathbb{N}^{*}$ that,

$$
\begin{aligned}
\widehat{u}_{n+1, r+1}-\widehat{u}_{n+1} \approx & \left(\widehat{u}_{n+1, r}-\widehat{u}_{n+1}\right)\left(\mathrm{i} \frac{\Delta t}{2}\left(\xi_{1}^{3}-\frac{\xi_{2}^{2}}{\xi_{1}} a-\frac{\xi_{3}^{2}}{\xi_{1}} b\right)\right) \\
& -\mathrm{i} \xi_{1} \frac{\Delta t}{2(p+1)} \sum_{j=0}^{p}\left(u_{n+1, r}-\widehat{\left.u_{n+1}\right)} u_{n+1, r}^{j} u_{n+1}^{p-j},\right.
\end{aligned}
$$

and a linearization (near $u_{n}$ ) in the right-hand side of this relation leads to the approximation:

$$
\widehat{u}_{n+1, r+1}-\widehat{u}_{n+1} \approx\left(\widehat{u}_{n+1, r}-\widehat{u}_{n+1}\right)\left(\mathrm{i} \frac{\Delta t}{2}\left(\xi_{1}^{3}-\frac{\xi_{2}^{2}}{\xi_{1}} a-\frac{\xi_{3}^{2}}{\xi_{1}} b\right)\right)-\mathrm{i} \xi_{1} \frac{\Delta t}{2}\left(u_{n+1, r}-u_{n+1}\right) u_{n}^{p} .
$$


Then, since $\xi_{1}=k \frac{\pi}{K}$, with $-\frac{N_{x}}{2} \leq k \leq \frac{N_{x}}{2}-1$, and $N_{x}=\frac{2 K}{\Delta x}$, it follows from similar considerations regarding $\xi_{2}, \xi_{3}$ that for $k \neq 0$, and making use of the Parseval formula, $\left\|u_{n+1, r+1}-u_{n+1}\right\|_{L^{2}(\Omega)} \lesssim \frac{\Delta t}{2} \frac{\pi}{\Delta x}\left(\frac{\pi^{2}}{(\Delta x)^{2}}+\frac{\Delta x K}{(\Delta y)^{2}}+\frac{\Delta x K}{(\Delta z)^{2}}+\left\|u_{n}\right\|_{L^{\infty}(\Omega)}^{p}\right)\left\|u_{n+1, r}-u_{n+1}\right\|_{L^{2}(\Omega)}$.

\section{Long time behavior for localized initial data}

We are interested here in numerical simulations concerning the Cauchy problem reformulated in (6) - (7). In a general way, two sets of parameters will be considered for these simulations based on the algorithm described previously. The first set is made up of the parameters $a, b= \pm 1$, $p \geq 1$, that intervene in (6), and the second one concerns the parameters $K, L, M, \Delta t, N_{x}, N_{y}$, $N_{z}$ used in the algorithm. Typically, after fixing $K, L$ and $M$, we affect values to the parameters $N_{x}, N_{y}, N_{z}$, and choose $\Delta t$ according to (10).

For studying the behavior in long time of the discrete solution of (2), associated with a localized initial datum, three phenomena will systematically be inspected: the dispersion (context where the $l^{\infty}$-norm of the solution decreases), the blow-up in finite time (situation where the $l^{\infty}$-norm of the solution tends to infinity), and the solitonic behavior (phenomenon where the $l^{\infty}$-norm of the solution is constant).

Our numerical inspections will be based on the choice:

$$
u_{0}(x, y, z)=\alpha\left(1-2 s x^{2}\right) \mathrm{e}^{-s\left(x^{2}+y^{2}+z^{2}\right)}
$$

and on various considerations of parameters mentioned above, including also the context ( $a=$ $b=-1, p \geq 2$ ) related to the known theoretical result (see J.-C. Saut [19]) of the blow-up in finite time. Let us indicate already that $\alpha, s \in \mathbb{R}_{+}^{*}$, and that the choices of values of $K$ will be such that $u_{0}$ satisfies (5) numerically.

In the presentation of our results, we distinguish the case $p=1$ and the generalized case where $p>1$.

\subsection{Non-generalized case: $p=1$}

In this subsection, we describe the results obtained from numerical simulations by fixing $p=1$.

\subsubsection{Evolution in long time}

In each of the contexts where $a= \pm 1$ with $b= \pm 1$, we perform experiments by setting here: $s=1, K=50, L=M=4, N_{y}=N_{z}=16$. 
We already mention that for all our experiments, here and in the next sections, the step $\Delta t$ will always be chosen in accordance with (10).

The results represented in Figures 1 and 2 concern the evolutions (with respect to time) of the $l^{\infty}$ and $l^{2}$-norms of the discrete solution and of the associated energy, obtained by considering $N_{x}=256, \Delta t=10^{-3}, \alpha=2$.
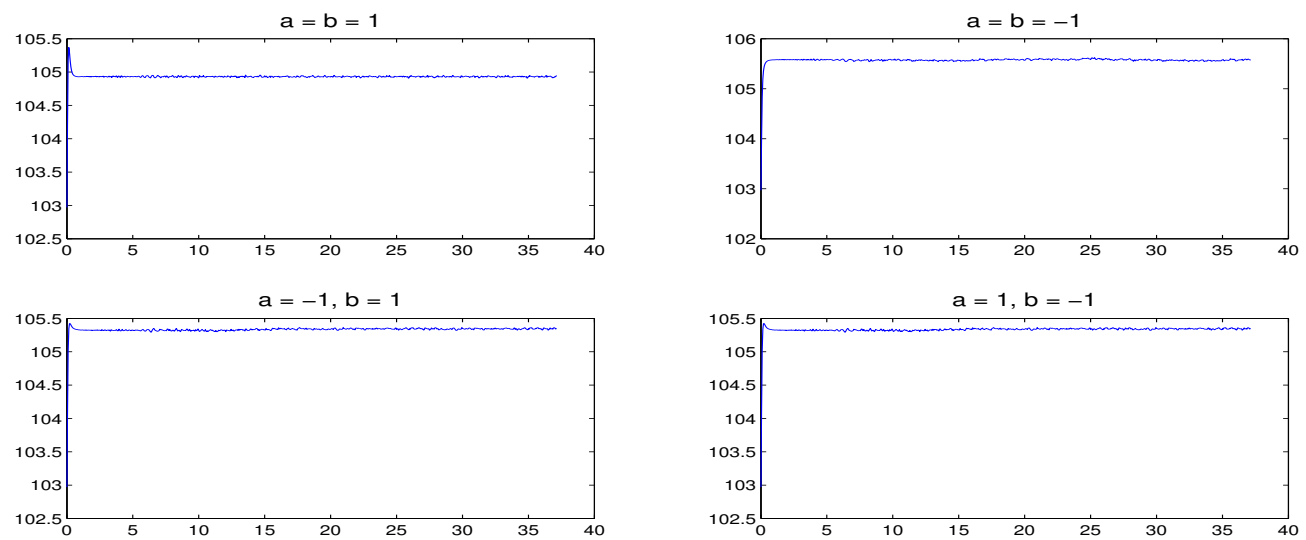

Figure 1: Evolutions of the energy with respect to time, for $p=1, \alpha=2, s=1, K=50, L=$ $M=4, N_{x}=256, N_{y}=N_{z}=16, \Delta t=10^{-3}$.
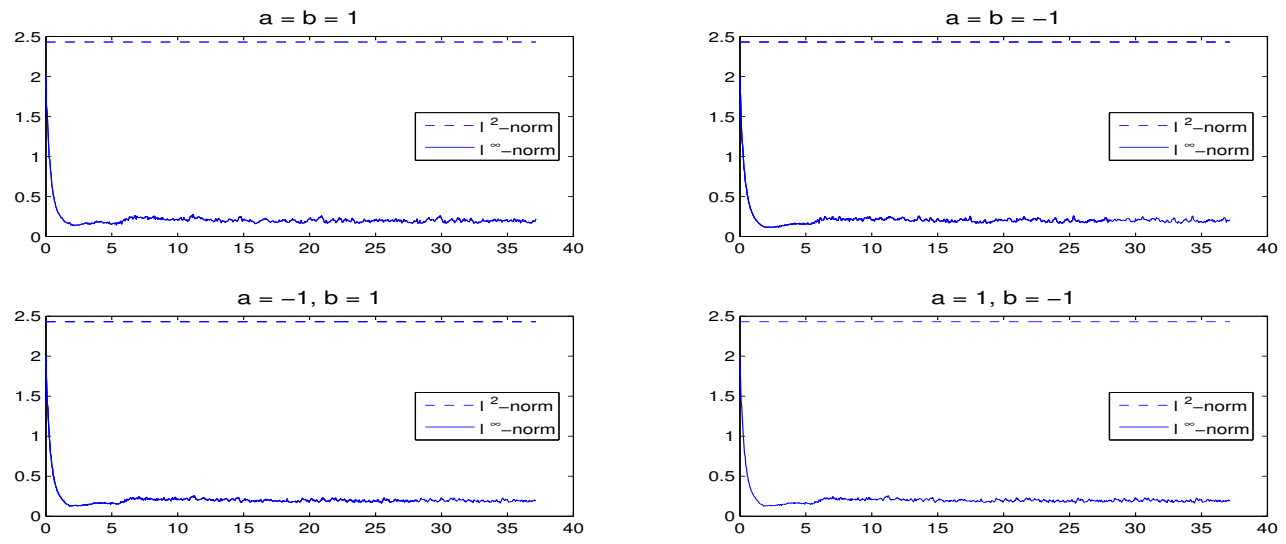

Figure 2: Evolutions of the $l^{2}-$ norm (---) and the $l^{\infty}$-norm (-) with respect to time, for $p=1, \alpha=2, s=1, K=50, L=M=4, N_{x}=256, N_{y}=N_{z}=16, \Delta t=10^{-3}$.

In particular, we observe the same evolutions of these norms and of the energy when $a=-1$, $b=1$ as well when $a=1, b=-1$. This was foreseeable according to the symmetry of $u_{0}$ and to the one of (2) related to the $y$ and $z$-directions. In this order of ideas, we restrict in this 
section the presentation of our results to the contexts where $a b=1$, and $a=-1, b=1$.

Figures 3 and 4 show also the evolutions of the $l^{\infty}$ and $l^{2}$-norms as well as of the energy, for the same values of the parameters, but now for $\alpha=5$. These evolutions are respectively similar to those observed when $\alpha=2$.
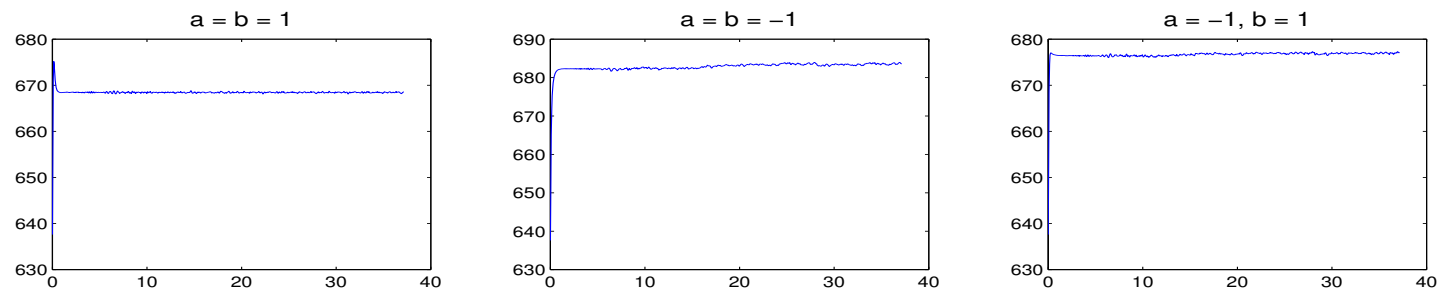

Figure 3: Evolutions of the energy with respect to time, for $p=1, \alpha=5, s=1, K=50, L=$ $M=4, N_{x}=256, N_{y}=N_{z}=16, \Delta t=10^{-3}$.
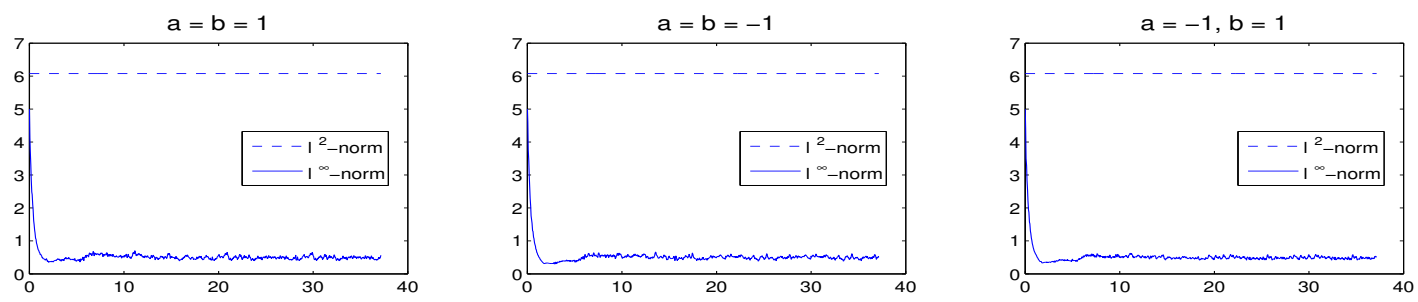

Figure 4: Evolutions of the $l^{2}-$ norm (---) and the $l^{\infty}$-norm (-) with respect to time, for $p=1, \alpha=5, s=1, K=50, L=M=4, N_{x}=256, N_{y}=N_{z}=16, \Delta t=10^{-3}$.

Let us notice that for these choices of $\alpha$, the results obtained from simulations with other values of parameters, namely with $K=32, L=M=6,12, N_{x}=128, N_{y}=N_{z}=32,64$, do not qualitatively differ from those represented in Figures 1 - 4. We observe that the dispersive effect prevails over the non-linearity. The evolutions of the norms in the different contexts where $a b= \pm 1$ look similar.

Figure 5 presents the evolutions of the $l^{\infty}$ and $l^{2}$-norms of the discrete solution when a larger amplitude of the initial datum is considered. Here $\alpha=50, N_{x}=512$ and $\Delta t=10^{-4}$.

In each of the contexts where $a=b=1, a b=-1$, we observe, as with the amplitudes $\alpha=2,5$, that the dispersive effect prevails over the non-linearity. But for $a=b=-1$, the discrete solution, associated with the initial datum of large amplitude, has a tendency to blow up at the beginning of the evolution and after that, the dispersive effect prevents the solution from blowing up. The fact that the non-linearity and the dispersive effect are neutralized, and that the $l^{\infty}$-norm is stabilized, is called the solitonic behavior. This means that the velocity of the limit profile has a periodic-like behavior in time. We mention that even with a time step, e.g. 

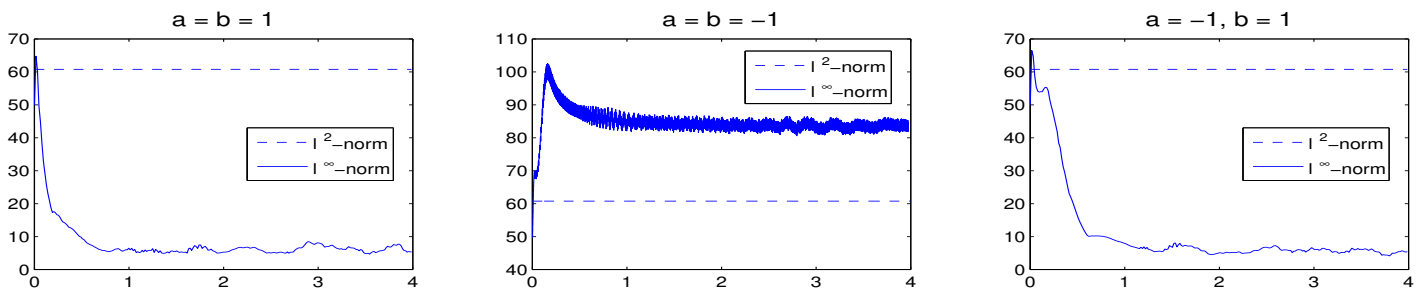

Figure 5: Evolutions of the $l^{2}$-norm (---) and the $l^{\infty}$-norm (-) with respect to time, for $p=1, \alpha=50, s=1, K=50, L=M=4, N_{x}=512, N_{y}=N_{z}=16, \Delta t=10^{-4}$.

$\Delta t=10^{-3}$, slightly bigger than the limiting value provided by (10) when we take larger values of $L, M, N_{y}, N_{z}$ (e.g. $L=M=6, N_{y}=N_{z}=32$ ), we do not obtain, from the simulations, results that are qualitatively different from those presented here.

\subsubsection{With a datum of larger $L^{2}-$ norm}

We now perform some experiments based on the initial datum (11) with a larger $L^{2}$-norm and a fixed amplitude. Thus, we consider $s=0.5$. Figures 6 and 7 present the evolutions of the $l^{\infty}$ and $l^{2}$-norms of the discrete solution as well as of the associated energy, obtained by using the same values of the parameters $\alpha, K, L, M, N_{x}, N_{y}, N_{z}$ and $\Delta t$ as in the experiments associated with Figures 3 and 4 , but with this new choice of $s$.
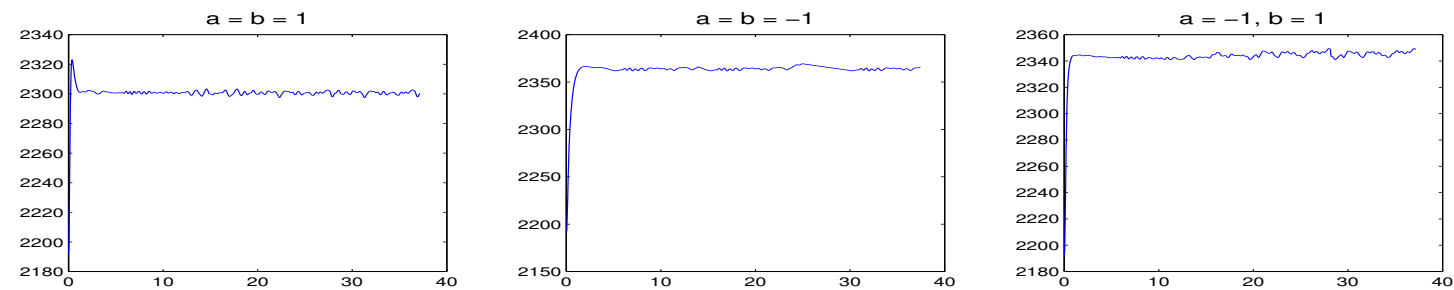

Figure 6: Evolutions of the energy with respect to time, for $p=1, \alpha=5, s=0.5, K=50, L=$ $M=4, N_{x}=256, N_{y}=N_{z}=16, \Delta t=10^{-3}$.
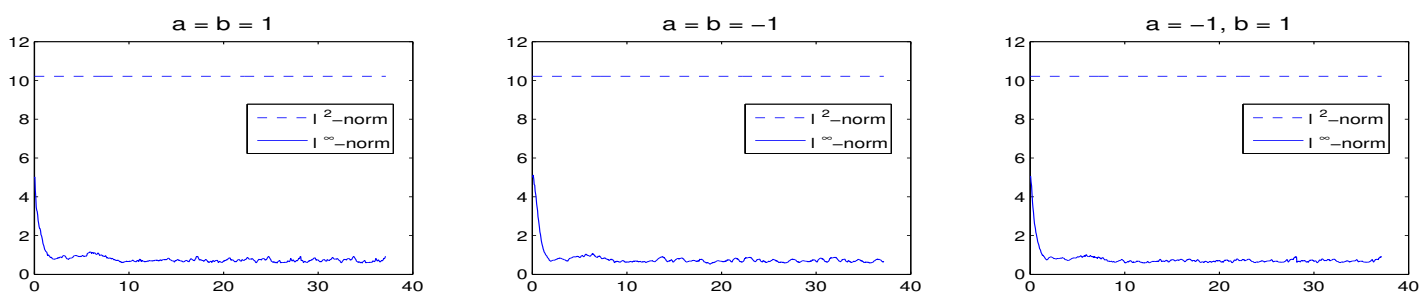

Figure 7: Evolutions of the $l^{2}$-norm (--) and the $l^{\infty}$-norm (-) with respect to time, for $p=1, \alpha=5, s=0.5, K=50, L=M=4, N_{x}=256, N_{y}=N_{z}=16, \Delta t=10^{-3}$. 
These results do not differ qualitatively from those that were represented in Figures 3 and 4, namely when $s=1$; there does not appear a qualitative influence of the $l^{2}$-norm of the initial datum on the evolution of the solution. Similar experiments have been performed, in this case where $s=0.5$, by considering $K=32, L=M=6, N_{x}=512, N_{y}=N_{z}=32, \Delta t=10^{-4}$ and a larger amplitude: $\alpha=50$. We notice the same observations as before; namely, the behavior of the solution is again governed by the dispersion in each of the contexts where $a b= \pm 1$, and a solitonic behavior is also observed in the context where $a=b=-1$.

\subsection{Generalized case: $p>1$}

In this subsection, we are concerned with numerical experiments when $p=2,3$. As mentioned before, a particular attention will be addressed to the contexts $(a=b=1, a b=-1)$ where no theoretical result has been established.

\subsubsection{The case $p=2$}

Let us fix in this part $p=2$. The experiments associated with Figures 8 - 10 are performed by setting: $s=1, K=50, L=M=4, N_{x}=256, N_{y}=N_{z}=16$ and $\Delta t=10^{-3}$. In Figures 8 and 9 , we represent the evolutions (with respect to time) of the $l^{\infty}$ and $l^{2}$-norms of the discrete solution and of the associated energy, obtained by considering $\alpha=2$.
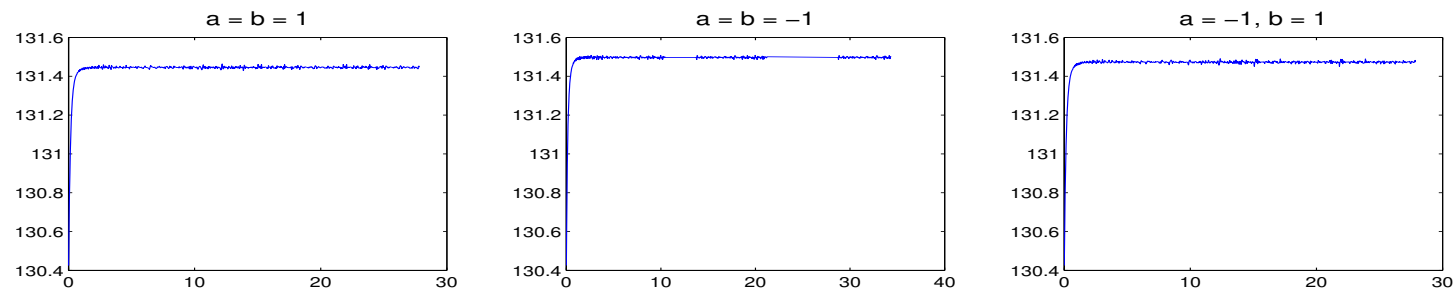

Figure 8: Evolutions of the energy with respect to time, for $p=2, \alpha=2, s=1, K=50, L=$ $M=4, N_{x}=256, N_{y}=N_{z}=16, \Delta t=10^{-3}$.
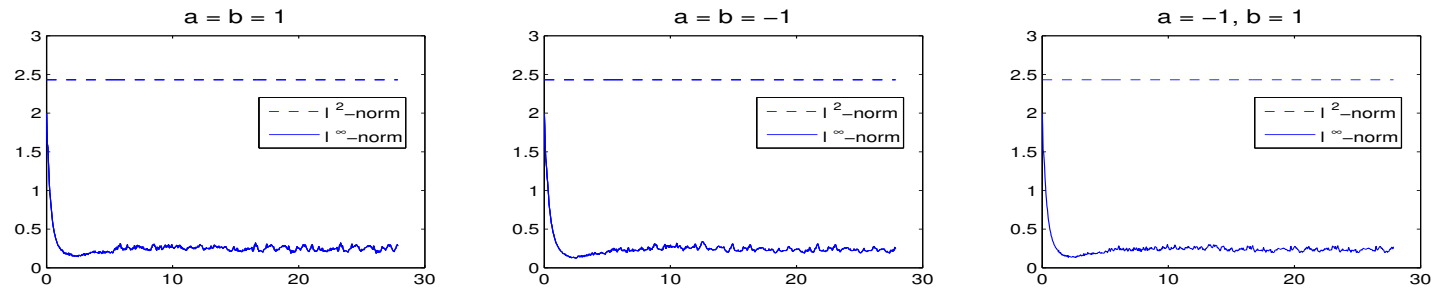

Figure 9: Evolutions of the $l^{2}$-norm (--) and the $l^{\infty}$-norm (-) with respect to time, for $p=2, \alpha=2, s=1, K=50, L=M=4, N_{x}=256, N_{y}=N_{z}=16, \Delta t=10^{-3}$. 
As for the case $p=1$, we observe that the dispersive effect prevails over the non-linearity. The evolutions of the norms in each of the contexts where $a b= \pm 1$ look similar. For this choice of $\alpha$, the same observations derive from simulations when we consider $K=32, L=M=6$, $N_{x}=128$, and $N_{y}=N_{z}=32$.

Figure 10 presents the results of the experiments performed by setting now $\alpha=5$.
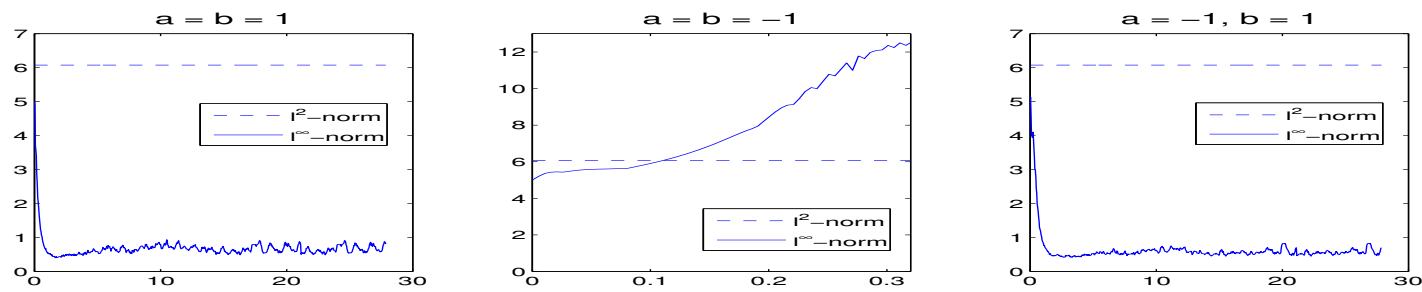

Figure 10: Evolutions of the $l^{2}-$ norm $(--)$ and the $l^{\infty}$-norm $(-)$ with respect to time, for $p=2, \alpha=5, s=1, K=50, L=M=4, N_{x}=256, N_{y}=N_{z}=16, \Delta t=10^{-3}$.

In each of the contexts where $a=b=1$ and $a b=-1$, we observe as previously that the dispersive effect prevails over the non-linearity. But in the context where $a=b=-1$, the behavior of the discrete solution looks different; the solution has a tendency to blow up at the beginning of the evolution. Let us refine these results by using a reduced time step as well as larger values for the parameters $N_{x}, N_{y}$ and $N_{z}$. In the experiments associated with Figure 11, we thus consider: $\Delta t=10^{-4}, N_{x}=512, N_{y}=N_{z}=32$, and $L=M=6$. It appears that the $l^{\infty}$-norm blows up in finite time and that the $l^{2}$-norm is consequently deteriorated from the blow-up instant.
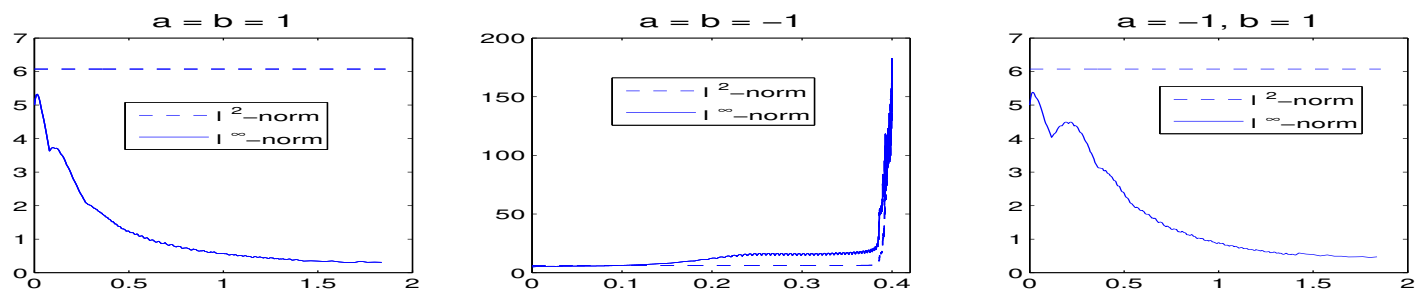

Figure 11: Evolutions of the $l^{2}-$ norm (--) and the $l^{\infty}$-norm (-) with respect to time, for $p=2, \alpha=5, s=1, K=50, L=M=6, N_{x}=512, N_{y}=N_{z}=32, \Delta t=10^{-4}$.

The theoretical result known from [19], for $p=2$, in the context where $a=b=-1$, is now exhibited numerically: the non-linearity is stronger than the dispersive effect and the solution blows up. The behavior of the solution is here completely different from the case where $p=1$. In the other contexts, the non-linearity tries to prevail over the dispersive effect at the beginning of the evolution but after that, the solution disperses completely. 
In order to study the influence of an initial datum of larger $l^{2}$-norm on the evolution of the solution, we use in the experiments associated with Figure 12 the same values of parameters as above, but set now $s=0.5$.
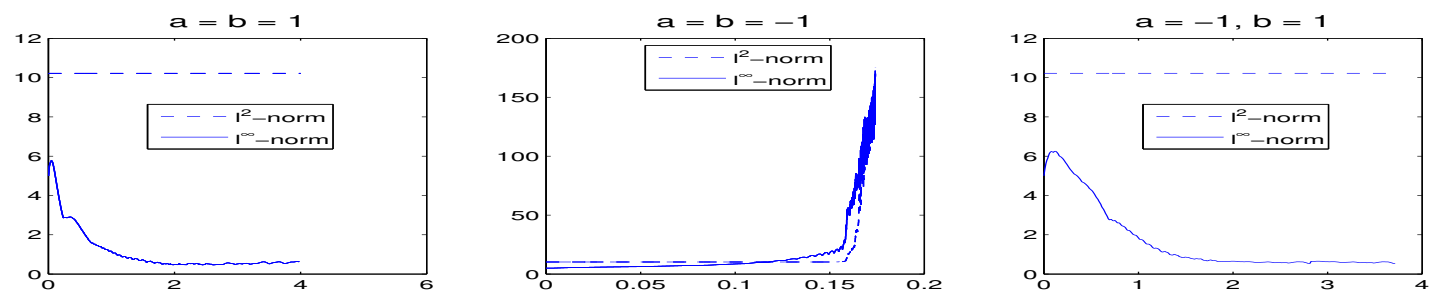

Figure 12: Evolutions of the $l^{2}-$ norm (--) and the $l^{\infty}$-norm (-) with respect to time, for $p=2, \alpha=5, s=0.5, K=50, L=M=6, N_{x}=512, N_{y}=N_{z}=32, \Delta t=10^{-4}$.

In the context where $a=b=-1$, the blow-up takes place earlier; the $l^{2}$-norm is consequently deteriorated from the blow-up instant. In the other contexts, there is no evidence of a qualitative influence of the $l^{2}$-norm of the initial datum on the evolution of the solution. In these contexts ( $a=b=1, a b=-1)$, where the dispersion seems to prevail against the non-linearity, let us inspect the effect of an initial datum of larger amplitude; we set now $\alpha=10, s=1$. In the experiments associated with Figure 13, we also consider $K=32, L=M=4, N_{x}=512$, $N_{y}=N_{z}=16, \Delta t=10^{-4}$.
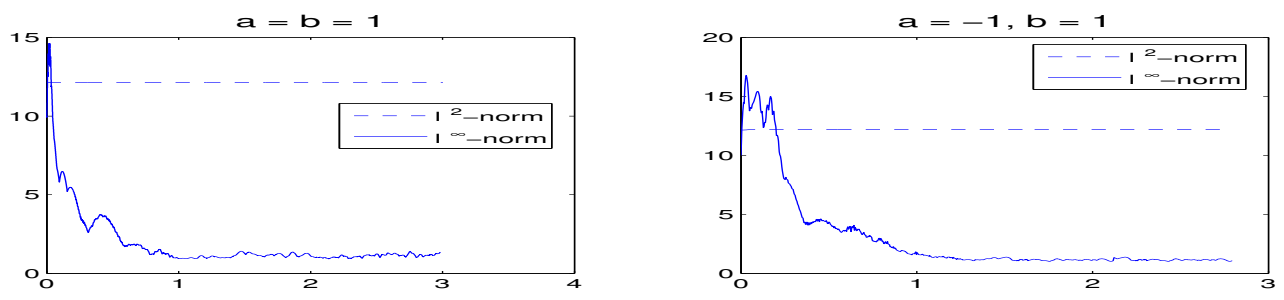

Figure 13: Evolutions of the $l^{2}$-norm (--) and the $l^{\infty}$-norm (-) with respect to time, for $p=2, \alpha=10, s=1, K=32, L=M=4, N_{x}=512, N_{y}=N_{z}=16, \Delta t=10^{-4}$.

The observations are the same as above, and are also confirmed when we use larger values of $N_{x}, N_{y}, N_{z}$ (e.g. with $N_{x}=1024, N_{y}=N_{z}=32$ ). Namely, in each of these three contexts, the solution has a tendency to blow up at the beginning of the evolution, but then disperses completely.

We also notice, in each of the contexts where $a b= \pm 1$, that the solution is subject to a more persistent non-linearity than when $p=1$. 


\subsubsection{The case $p=3$}

We can already predict that, since the non-linearity was persistent with $p=2$, the blow-up in finite time will be observed in the case $p=3$ because of a stronger non-linearity.

Let us now fix $p=3$. In our first experiments, associated with Figures $14-16$, we have considered $s=1, L=M=4, N_{x}=256, N_{y}=N_{z}=16$ and $\Delta t=10^{-3}$. Figures 14 and 15 show the evolutions (with respect to time) of the $l^{\infty}$ and $l^{2}$-norms of the discrete solution, and of the associated energy, obtained when $K=50, \alpha=2$.
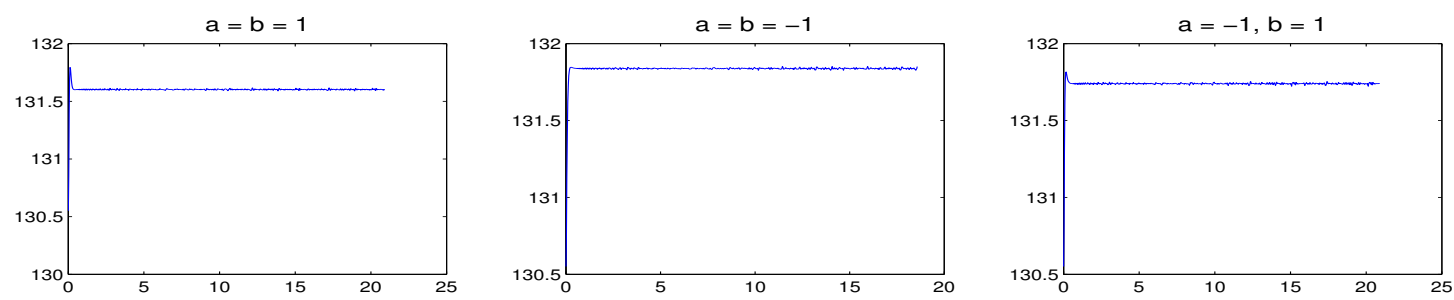

Figure 14: Evolutions of the energy with respect to time, for $p=3, \alpha=2, s=1, K=50, L=$ $M=4, N_{x}=256, N_{y}=N_{z}=16, \Delta t=10^{-3}$.
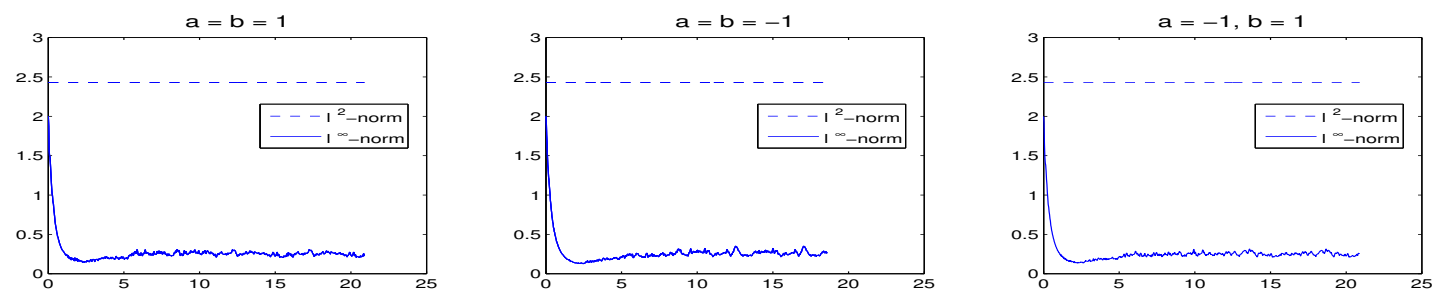

Figure 15: Evolutions of the $l^{2}$-norm (--) and the $l^{\infty}$-norm (-) with respect to time, for $p=3, \alpha=2, s=1, K=50, L=M=4, N_{x}=256, N_{y}=N_{z}=16, \Delta t=10^{-3}$.

The evolutions of the norms, in each of the contexts where $a b= \pm 1$, look similar, and as for $p=1,2$, the dispersive effect prevails over the non-linearity. Similar observations derive from simulations when using other values of parameters, namely with $\Delta t=10^{-4}, K=32$, $L=M=6,8, N_{x}=512, N_{y}=N_{z}=32,64$.

Figure 16 shows the results obtained by considering now a slightly larger amplitude: $\alpha=3$. 

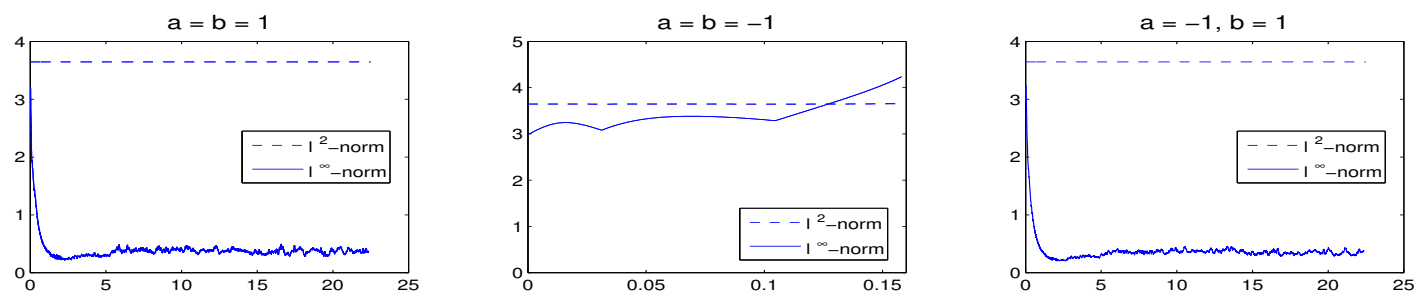

Figure 16: Evolutions of the $l^{2}-$ norm $(--)$ and the $l^{\infty}$-norm $(-)$ with respect to time, for $p=3, \alpha=3, s=1, K=32, L=M=4, N_{x}=256, N_{y}=N_{z}=16, \Delta t=10^{-3}$.

The observations remain the same as above, in each of the contexts where $a=b=1$, and $a b=-1$. However, in the context where $a=b=-1$, the solution has a tendency to blow up. Let us refine the results by using larger values for $L, M, N_{x}, N_{y}, N_{z}$ and reducing $\Delta t$. We take $L=M=6, N_{x}=512, N_{y}=N_{z}=32, \Delta t=10^{-4}$, and keep the same values for $K, \alpha, s$ as in the experiments associated with Figure 16.
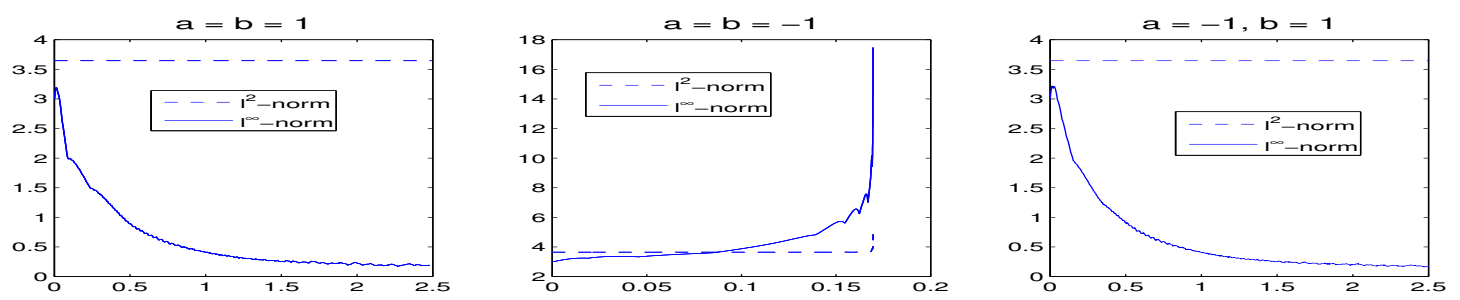

Figure 17: Evolutions of the $l^{2}$-norm (--) and the $l^{\infty}$-norm (-) with respect to time, for $p=3, \alpha=3, s=1, K=32, L=M=6, N_{x}=512, N_{y}=N_{z}=32, \Delta t=10^{-4}$.

These experiments confirm, in the context where $a=b=-1$, that the non-linearity is effectively stronger than the dispersive effect. In the other contexts, where the dispersive effect seems to prevail over the non-linearity, let us inspect the influence of a slightly larger amplitude; we thus consider $\alpha=4$ in the experiments associated with Figure 18.
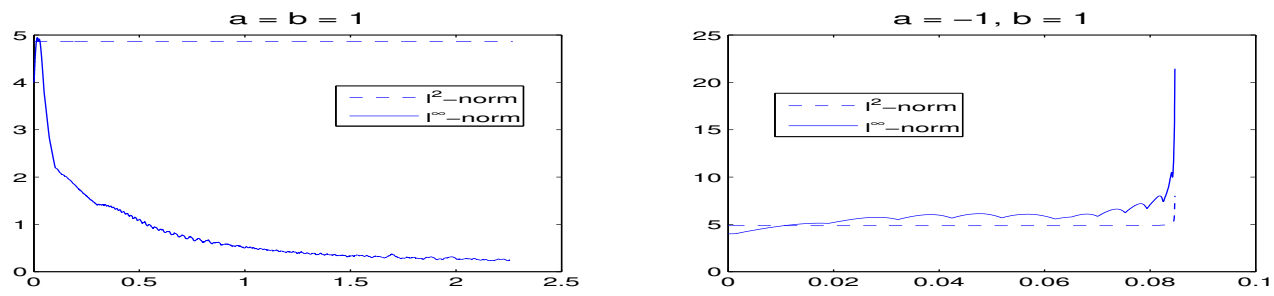

Figure 18: Evolutions of the $l^{2}$-norm (--) and the $l^{\infty}$-norm (-) with respect to time, for $p=3, \alpha=4, s=1, K=32, L=M=6, N_{x}=512, N_{y}=N_{z}=32, \Delta t=10^{-4}$. 
The results now show that the non-linearity is stronger than the dispersive effect in each of the contexts where $a b=-1$; the solution blows up in these contexts. Of course, the $l^{2}$-norm is consequently deteriorated from the blow-up instant.

When we inspect nearer the context where $a=b=1$, by considering again a more large amplitude, $\alpha=5$, we obtain the results represented in Figure 19 that allow us to notice that the solution blows up also in this context. The energy as well as the $l^{2}$-norm are consequently deteriorated from the blow-up instant. This blow-up of the solution, for positive transverse directions, reminds us of the numerical result presented by F. Hamidouche [9] in the context of the generalized KP-II equation for which, when $p=3$, the solution blows up for an initial datum of large amplitude.
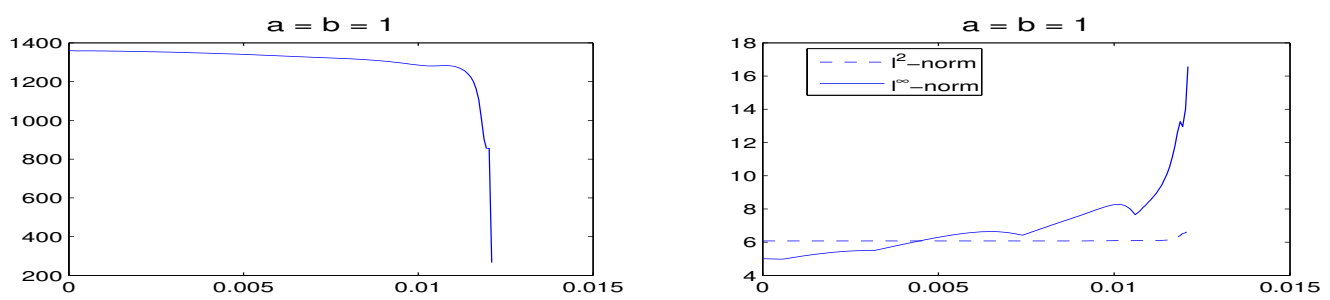

Figure 19: Evolutions of the energy at left, of the $l^{2}$-norm (--) and of the $l^{\infty}$-norm (-) at right, with respect to time. Here, $p=3, \alpha=5, s=1, K=32, L=M=6, N_{x}=512, N_{y}=$ $N_{z}=32, \Delta t=10^{-4}$.

The experiments associated with Figure 20, and performed by setting $s=0.5$, allow us to study the influence of an initial datum of larger $l^{2}$-norm.
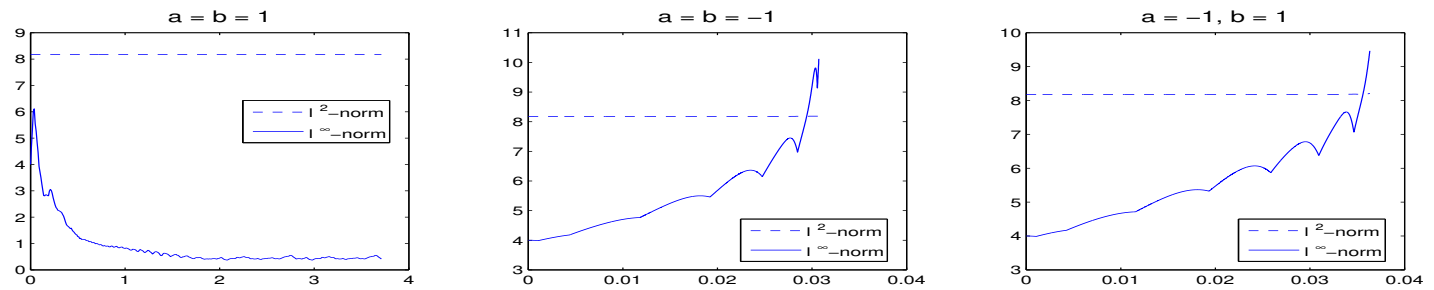

Figure 20: Evolutions of the $l^{2}$-norm (--) and the $l^{\infty}$-norm (-) with respect to time, for $p=3, \alpha=4, s=0.5, K=32, L=M=6, N_{x}=512, N_{y}=N_{z}=32, \Delta t=10^{-4}$. 
The evolution of the solution, in the context where $a=b=1$, seems to not be influenced in a qualitative way by such an initial datum. However, in each of the contexts where $a=b=-1$, and $a b=-1$, the blow-up takes place earlier.

Let us now summarize our results. The effect of two negative transverse directions is much less regularizing than the effect of two positive or "opposite" transverse directions. Indeed, the discrete solution, in the context where $a=b=-1$, blows up in finite time when $p=2$, whereas the one involved in each of the contexts where $a=b=1$, and $a b=-1$, blows up in finite time when $p=3$.

The results obtained in the case where $p=3$ lead us to formulate the following conjecture.

\section{Conjecture 3.1}

Let $p \geq 3$. If $a=b=1$ or $a b=-1$, then there exist localized initial data for which the solutions of (2) blow up in finite times.

\section{Transverse instabilities in the $y$ and $z$-directions}

The generalized equation (2) admits as solution the line-soliton

$$
\Phi_{c}(x, t)=\left(\frac{(p+1)(p+2)}{2} c\right)^{1 / p} \operatorname{sech}^{2 / p}\left(\frac{p \sqrt{c}}{2}(x-c t)\right),
$$

which is a solution of the generalized KdV equation. This represents a solitary wave, moving from left to right in the $x$-direction, at velocity $c$, without deformation. In this section, we study the evolution in time of this line-soliton perturbed in an infinitesimal way in the two transverse $y$ and $z$-directions. We consider then the Cauchy problem reformulated in (3), by defining the initial datum $u_{0}$ as follows,

$$
u_{0}(x, y, z):=\Phi_{c(y, z)}(x, 0)
$$

where $c(y, z):=c\left(1+\varepsilon \cos \left(\frac{2 \pi y}{\lambda_{y}}+\frac{2 \pi z}{\lambda_{z}}\right)\right)$, with $\varepsilon>0$ a fixed small value; this perturbed velocity is not uniform in the transverse directions, $\lambda_{y}$ and $\lambda_{z}$ are the wavelengths.

\subsection{Non-generalized case: $p=1$}

We fix $p=1$ in this subsection. In addition to the two previous sets of parameters, our numerical experiments will be performed with respect to diverse considerations of $c, \lambda_{y}$ and $\lambda_{z}$. 


\subsubsection{Evolution in long time}

In each of the contexts where $a b= \pm 1$, we perform simulations by fixing $K=32, L=M=4$, $N_{x}=256, N_{y}=N_{z}=16, \Delta t=5 \times 10^{-4}$. Also, we start by considering $\varepsilon=10^{-2}$ and the context where $a=b=-1$.

In Figure 21, we represent the evolutions of the $l^{2}$ and $l^{\infty}$-norms of the discrete solution obtained respectively with $\lambda_{y}=\lambda_{z}=0.01,2,2.1$ and 50 , for $c=2$.
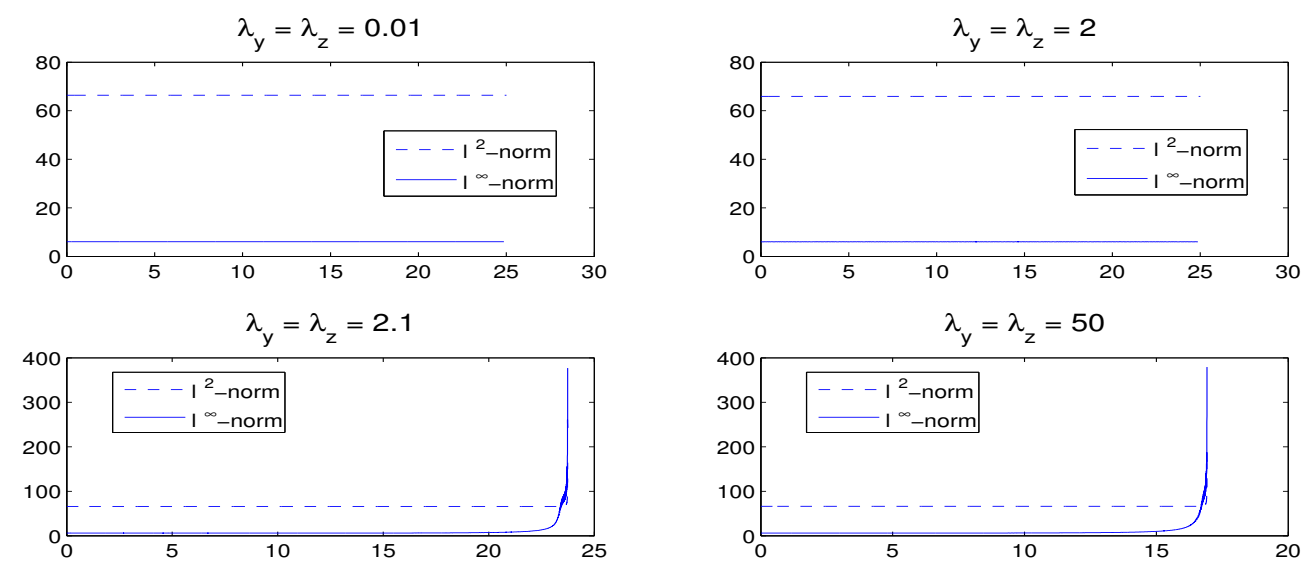

Figure 21: Evolutions of the $l^{2}$-norm (--) and the $l^{\infty}$-norm $(-)$ with respect to time, for $p=1, a=b=-1, c=2, K=32, L=M=4, N_{x}=256, N_{y}=N_{z}=16, \Delta t=5 \times 10^{-4}$. Here, $\lambda_{y}=\lambda_{z}=0.01,2,2.1,50$, and $\varepsilon=10^{-2}$.

It seems that for $\lambda_{y}$ and $\lambda_{z}$ bigger than $\lambda^{1}$, where $\lambda^{1} \simeq 2.1$, the perturbed line-soliton blows up. For such wavelengths, the $l^{\infty}$-norm blows up in finite time, and the $l^{2}$-norm is consequently deteriorated from the blow-up instant. We notice that the larger the wavelengths are, the earlier the blow-up occurs. Similar results derive also from simulations by using larger values for $K, L, M, N_{x}, N_{y}, N_{z}$ (e.g. with $K=50, L=M=6, N_{x}=512, N_{y}=N_{z}=32$ ) even with a slightly bigger time step, e.g. $\Delta t=10^{-3}$.

In a general way and about the results of this section, let us mention that in presence of the blow-up of the $l^{\infty}$-norm, a deterioration of the $l^{2}$-norm, from the blow-up instant, will consequently be observed.

Other experiments are performed by considering now distinct values for $\lambda_{y}$ and $\lambda_{z}$. Figure 22 presents the results obtained with $\lambda_{y}=0.01,2$ and $\lambda_{z}=2.1,50$. 

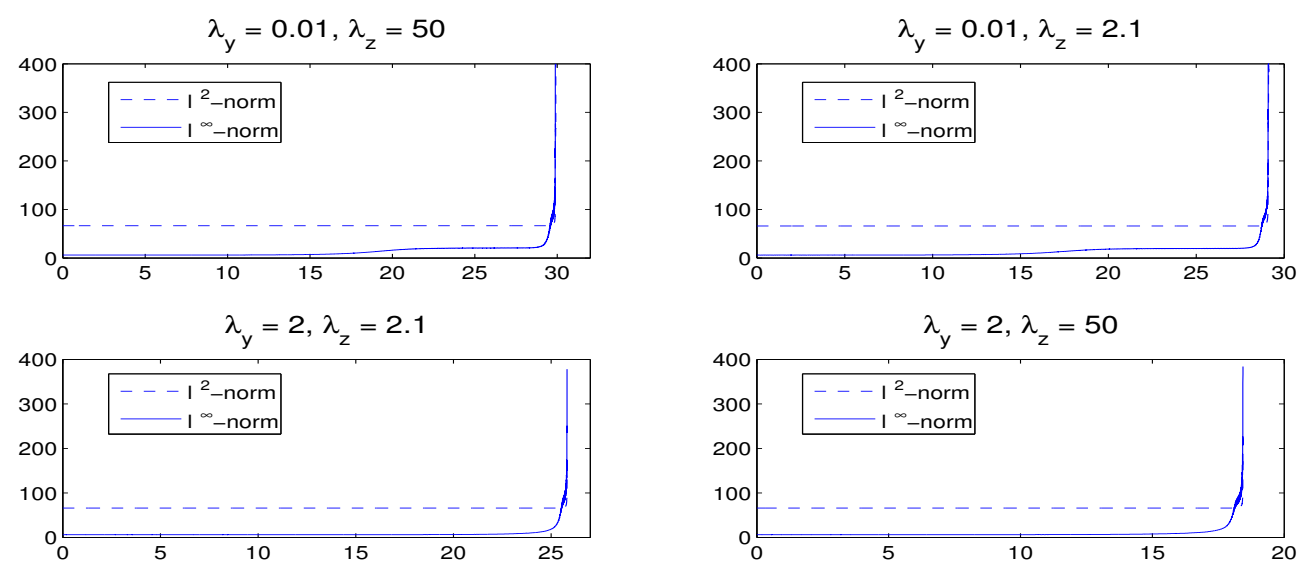

Figure 22: Evolutions of the $l^{2}$-norm (---) and the $l^{\infty}$-norm (-) with respect to time, for $p=1, a=b=-1, c=2, K=32, L=M=4, N_{x}=256, N_{y}=N_{z}=16, \Delta t=5 \times 10^{-4}$. Here, $\lambda_{y}=0.01,2, \lambda_{z}=2.1,50$ and $\varepsilon=10^{-2}$.

There exists a critical transverse wavelength $\lambda_{2}^{1} \simeq 2.1$ such that if $\lambda_{y}$ or $\lambda_{z}$ is larger than $\lambda_{2}^{1}$, the perturbed line-soliton blows up.

We want now to inspect the influence of the velocity as regards this observation. The change of functions

$$
\widetilde{u}(x, y, z, t):=\frac{1}{c} u\left(\frac{x}{\sqrt{c}}, \frac{y}{c}, \frac{z}{c}, \frac{t}{c \sqrt{c}}\right),
$$

applied to (2), for $p=1$, gives

$$
\widetilde{u}_{t}+\widetilde{u} \widetilde{u}_{x}+\widetilde{u}_{x x x}+a \partial_{x}^{-1} \widetilde{u}_{y y}+b \partial_{x}^{-1} \widetilde{u}_{z z}=0
$$

The initial datum becomes

$$
\widetilde{u}_{0}(x, y, z)=3\left(1+\varepsilon \cos \left(\frac{2 \pi y}{c \lambda_{y}}+\frac{2 \pi z}{c \lambda_{z}}\right)\right) \operatorname{sech}\left(\frac{x}{2} \sqrt{1+\varepsilon \cos \left(\frac{2 \pi y}{c \lambda_{y}}+\frac{2 \pi z}{c \lambda_{z}}\right)}\right) .
$$

We then notice that propagating a line-soliton of velocity $c$ perturbed in the two transverse directions by the wavelengths $\lambda_{y}$ and $\lambda_{z}$ is equivalent to propagating a line-soliton of velocity equal to 1 perturbed in the two transverse directions by the wavelengths $c \lambda_{y}$ and $c \lambda_{z}$.

The experiments with $c=2$ show that there exists a critical value $\lambda_{2}^{1}(\simeq 2.1)$ that indicates an "instability condition". Of course, from the previous change of functions, if $\lambda_{y}$ or $\lambda_{z}$ is larger than $\lambda_{c}^{1}:=\frac{4.2}{c}$, then the perturbed line-soliton blows up and is hence unstable.

Let us now confirm this indication by taking $c=4$. Figure 23 presents the results obtained by considering then identical wavelengths: $\lambda_{y}=\lambda_{z}=0.01,1,1.1,50$. 

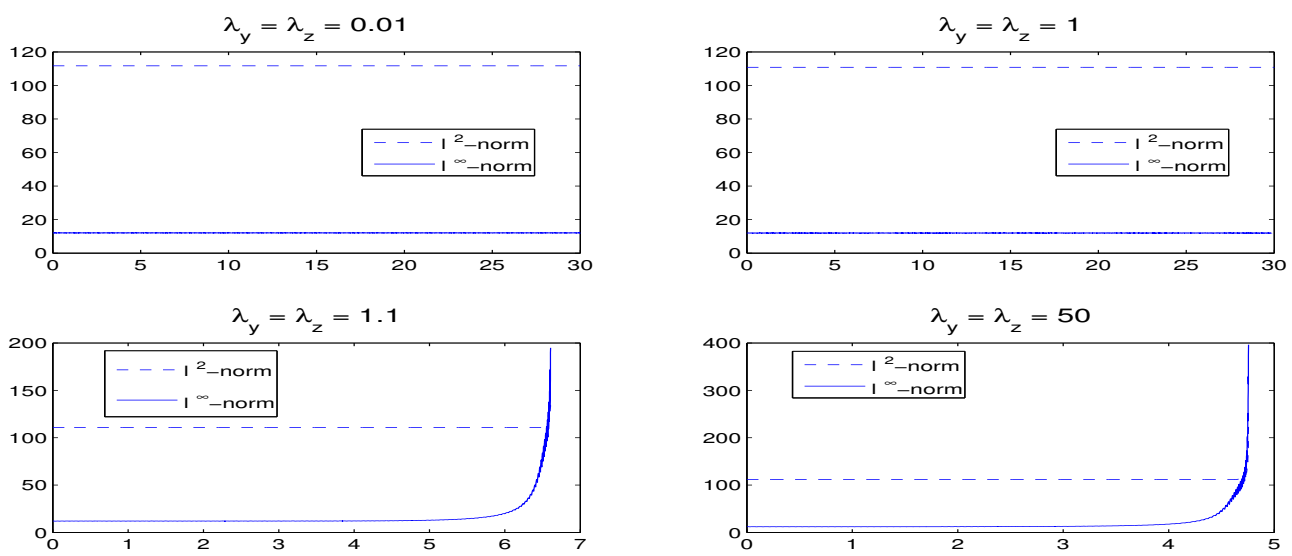

Figure 23: Evolutions of the $l^{2}$-norm (---) and the $l^{\infty}$-norm (-) with respect to time, for $p=1, a=b=-1, c=4, K=32, L=M=4, N_{x}=256, N_{y}=N_{z}=16, \Delta t=5 \times 10^{-4}$. Here, $\lambda_{y}=\lambda_{z}=0.01,1,1.1,50$ and $\varepsilon=10^{-2}$.

By contrast, the experiments associated with Figure 24 consider distinct wavelengths: $\lambda_{y}=$ $0.01,1$ and $\lambda_{z}=1.1,50$.
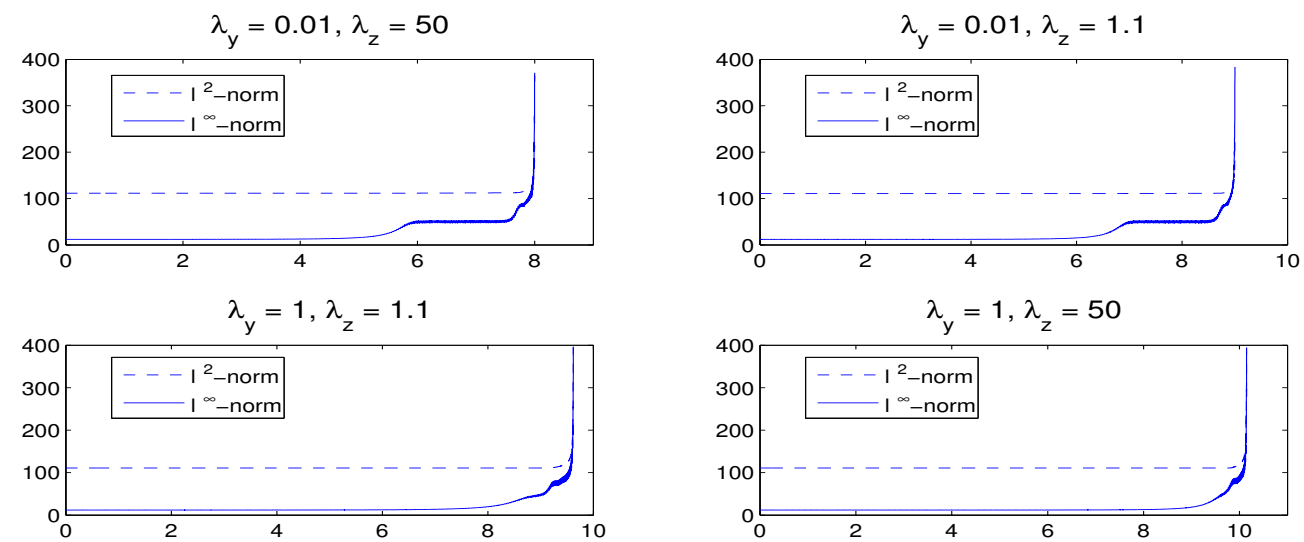

Figure 24: Evolutions of the $l^{2}$-norm (--) and the $l^{\infty}$-norm (-) with respect to time, for $p=1, a=b=-1, c=4, K=32, L=M=4, N_{x}=256, N_{y}=N_{z}=16, \Delta t=5 \times 10^{-4}$. Here, $\lambda_{y}=0.01,1, \lambda_{z}=1.1,50$ and $\varepsilon=10^{-2}$.

The results represented in Figures 23 and 24 lead us to the observations that were also reported for $c=2$.

We propose to extend our experiments to the case of very large wavelengths in order to inspect numerically the following aspect: when $\lambda_{y}$ and $\lambda_{z}$ tend to infinity, the formal limit of the perturbed line-soliton gives $\Phi_{c(1+\varepsilon)}$, which does not depend on the transverse variables. In 
particular, the solution of (2) with $\Phi_{c(1+\varepsilon)}$ as initial datum is stable [2].

Figure 25 presents the results obtained by considering then $\lambda_{y}=1,1.1,10^{8}, 10^{9}$ and $\lambda_{z}=$ $10^{8}, 10^{9}$.
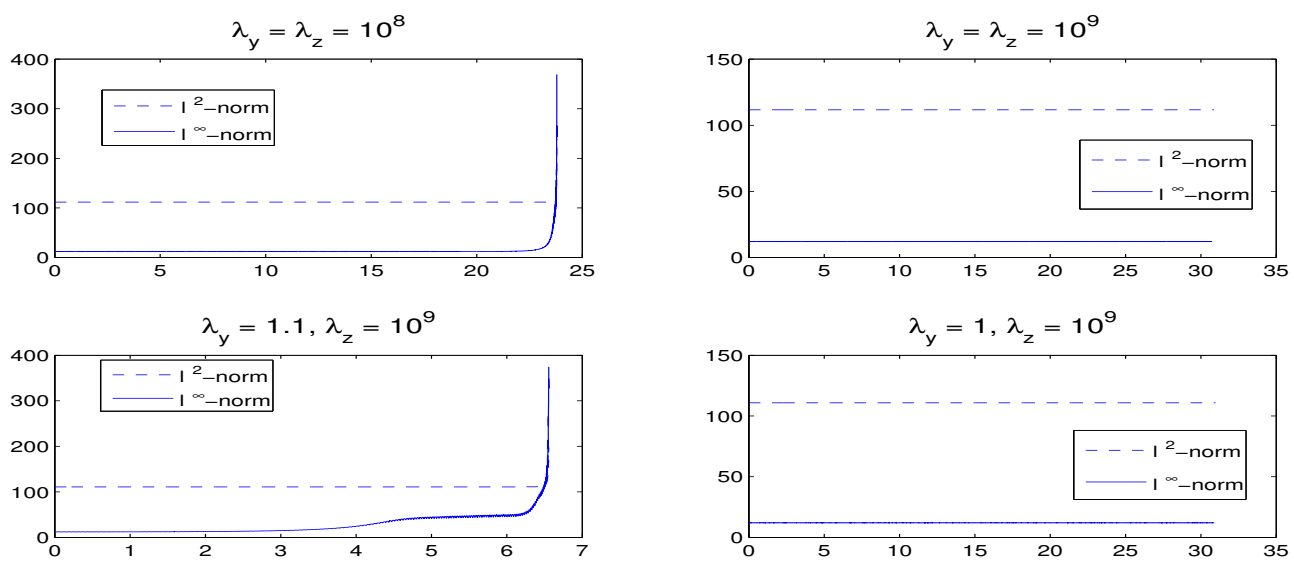

Figure 25: Evolutions of the $l^{2}$-norm (--) and the $l^{\infty}$-norm (-) with respect to time, for $p=1, a=b=-1, c=4, K=32, L=M=4, N_{x}=256, N_{y}=N_{z}=16, \Delta t=5 \times 10^{-4}$. Here, $\lambda_{y}=1,1.1,10^{8}, 10^{9}, \lambda_{z}=10^{8}, 10^{9}$ and $\varepsilon=10^{-2}$.

We thus deduce, in the context where $a=b=-1$, that there exists another critical transverse wavelength $\lambda_{c}^{2}$ such that if $\lambda_{y}, \lambda_{z}>\lambda_{c}^{2}$, the perturbed line-soliton no longer blows up.

Let us now consider the contexts where $a b=-1$, by using again $c=4$ in the experiments. Figures 26 and 27 show the results obtained respectively for $a=1, b=-1$ and $a=-1, b=1$, when $\lambda_{y}=\lambda_{z}=0.01,1.1,50$ and $\lambda_{y}=0.01$ with $\lambda_{z}=50$.
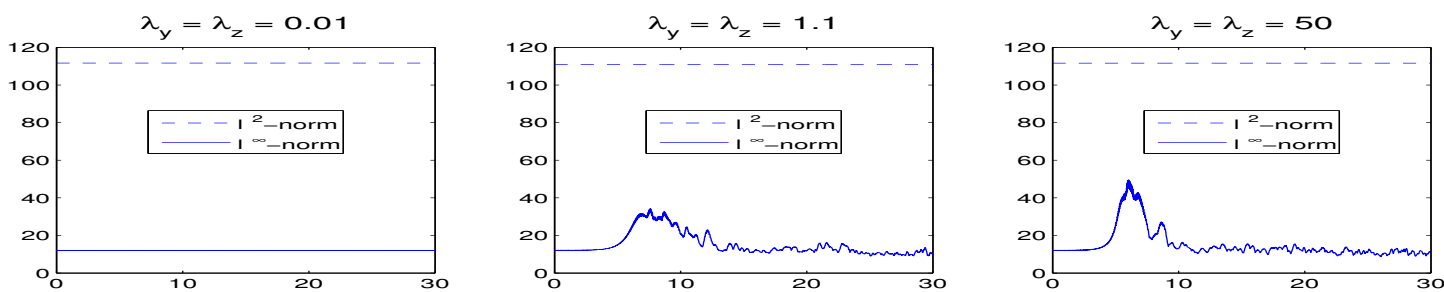

Figure 26: Evolutions of the $l^{2}-$ norm (--) and the $l^{\infty}$-norm (-) with respect to time, for $p=1, a=-1, b=1, c=4, K=32, L=M=4, N_{x}=256, N_{y}=N_{z}=16, \Delta t=5 \times 10^{-4}$. Here $\lambda_{y}=\lambda_{z}=0.01,1.1,50$ and $\varepsilon=10^{-2}$.

We observe from simulations the same evolutions of the norms in the contexts where $a b=-1$, and when $\lambda_{y}=\lambda_{z}$. This was foreseeable due to the symmetry of $u_{0}$ (in that case) and to the one of (2) related to the $y$ and $z$-directions. 

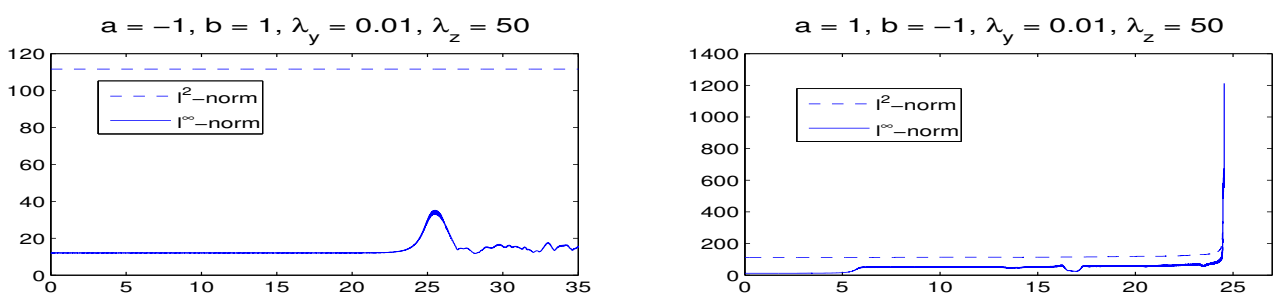

Figure 27: Evolutions of the $l^{2}$-norm (--) and the $l^{\infty}$-norm (-) with respect to time, for $p=1, a b=-1, c=4, K=32, L=M=4, N_{x}=256, N_{y}=N_{z}=16, \Delta t=5 \times 10^{-4}$. Here $\lambda_{y}=0.01, \lambda_{z}=50$ and $\varepsilon=10^{-2}$.

The experiments associated with Figure 28 are performed in the context of very large values of wavelengths, and for $a b=-1$; here also, the same evolutions of the norms are observed when $\lambda_{y}=\lambda_{z}$. This is not the case when $\lambda_{y} \neq \lambda_{z}$ as, in particular, indicate Figures 27 and 28.
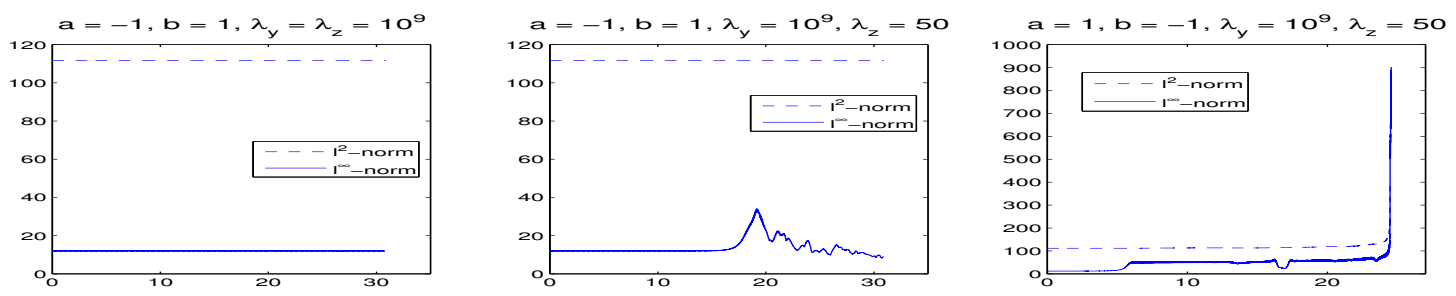

Figure 28: Evolutions of the $l^{2}$-norm (--) and the $l^{\infty}$-norm (-) with respect to time, for $p=1, a b=-1, c=4, K=32, L=M=4, N_{x}=256, N_{y}=N_{z}=16, \Delta t=5 \times 10^{-4}$. Here $\lambda_{y}=10^{9}, \lambda_{z}=50,10^{9}$ and $\varepsilon=10^{-2}$.

We notice in the context where $a=1, b=-1$, respectively $a=-1, b=1$, a tendency of blow-up when $\lambda_{c}^{1}<\lambda_{y}<\lambda_{c}^{2}$, respectively $\lambda_{c}^{1}<\lambda_{z}<\lambda_{c}^{2}$ (see Figures 26 - 28); it seems that another form of stability has appeared (see e.g. the cases $\lambda_{y}=\lambda_{z}=1.1,50$ in Figure 26). On the other hand, when $\lambda_{y}<\lambda_{c}^{1}$, or $\lambda_{y}>\lambda_{c}^{2}$, and $\lambda_{c}^{1}<\lambda_{z}<\lambda_{c}^{2}$ (respectively $\lambda_{z}<\lambda_{c}^{1}$, or $\lambda_{z}>\lambda_{c}^{2}$, and $\lambda_{c}^{1}<\lambda_{y}<\lambda_{c}^{2}$ ), where $0<\lambda_{c}^{1} \ll \lambda_{c}^{2}$ are critical values depending only on $c$, the corresponding solution blows up. These observations can also be explained partly by the fact that the solution of KP-I with a line-soliton uniquely perturbed in the $y$-direction, as initial datum, is unstable for a sufficiently large wavelength. We indicate that even for a larger velocity, e.g. with $c=5$, the same observations derive from the simulations.

The experiments associated with Figure 29 consider the last situation, $a=b=1$, and are also performed for $c=4$. Here, the perturbed line-soliton seems to never blow up. 

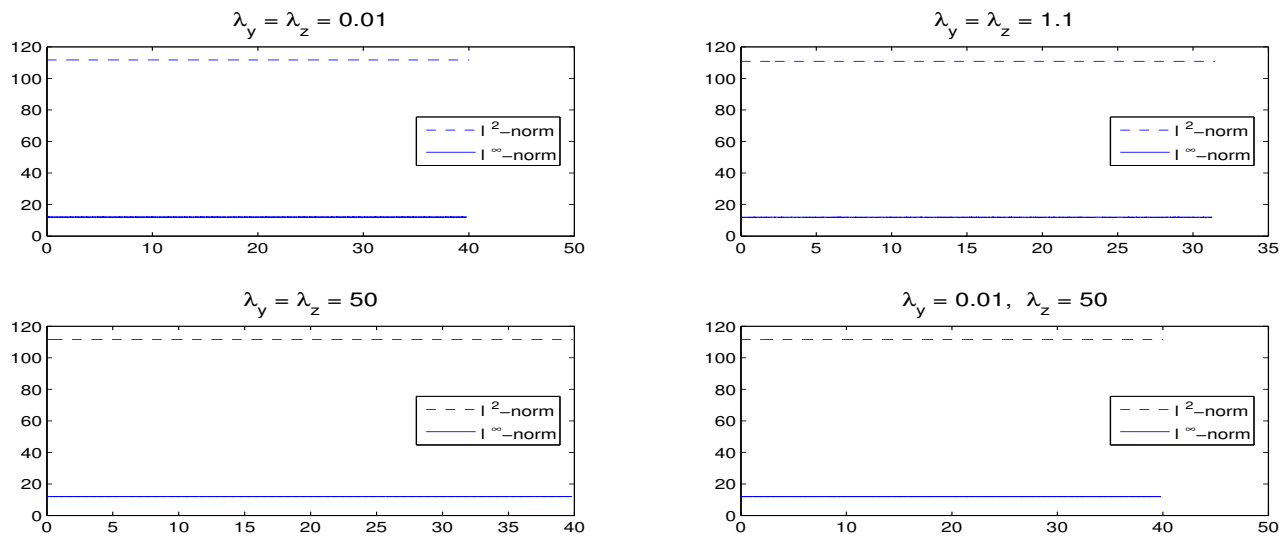

Figure 29: Evolutions of the $l^{2}$-norm (---) and the $l^{\infty}$-norm (-) with respect to time, for $p=1, a=b=1, c=4, K=32, L=M=4, N_{x}=256, N_{y}=N_{z}=16, \Delta t=5 \times 10^{-4}$. Here, $\lambda_{y}=\lambda_{z}=0.01,1.1,50, \lambda_{y}=0.01, \lambda_{z}=50$ and $\varepsilon=10^{-2}$.

We want now to study the possible influence of a smaller value of $\varepsilon$ on the observations noticed previously. We thus set: $\varepsilon=5 \times 10^{-3}$. In order to compare the results represented in Figures 23, 26 and 27 with those that will be obtained here, we use in the present experiments the new value of $\varepsilon$, and the same values that were affected to the other parameters for providing these previous results. Figures 30 and 31 present the results obtained in the context where $a=b=-1$. Contrary to the experiments associated with Figure 30, those associated with Figure 31 consider very large wavelengths.
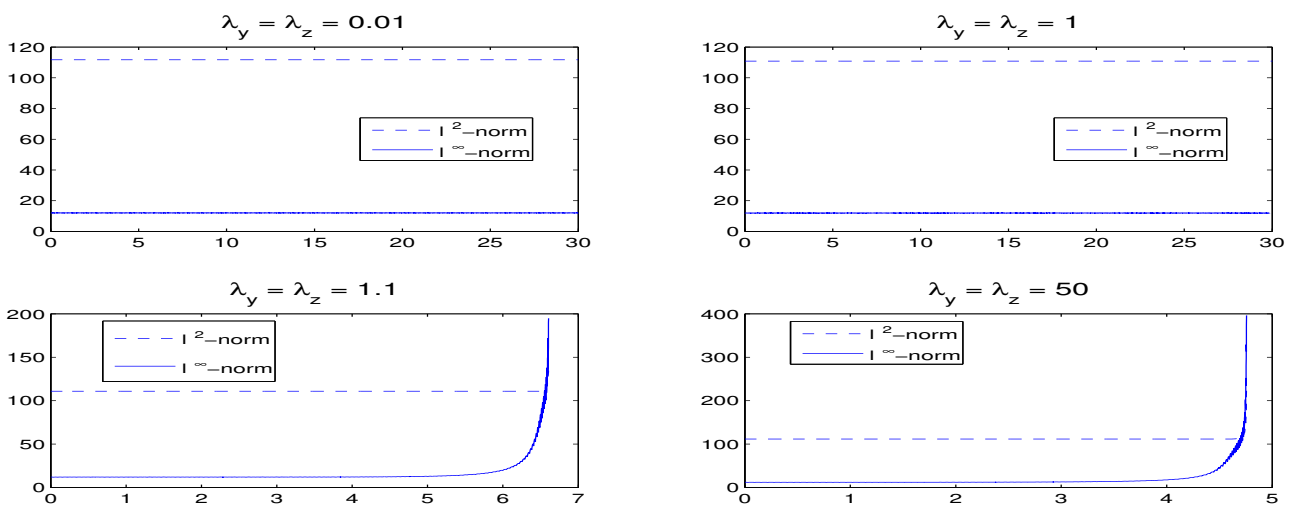

Figure 30: Evolutions of the $l^{2}$-norm (--) and the $l^{\infty}$-norm (-) with respect to time, for $p=1, a=b=-1, c=4, K=32, L=M=4, N_{x}=256, N_{y}=N_{z}=16, \Delta t=5 \times 10^{-4}$. Here, $\lambda_{y}=\lambda_{z}=0.01,1,1.1,50$ and $\varepsilon=5 \times 10^{-3}$. 

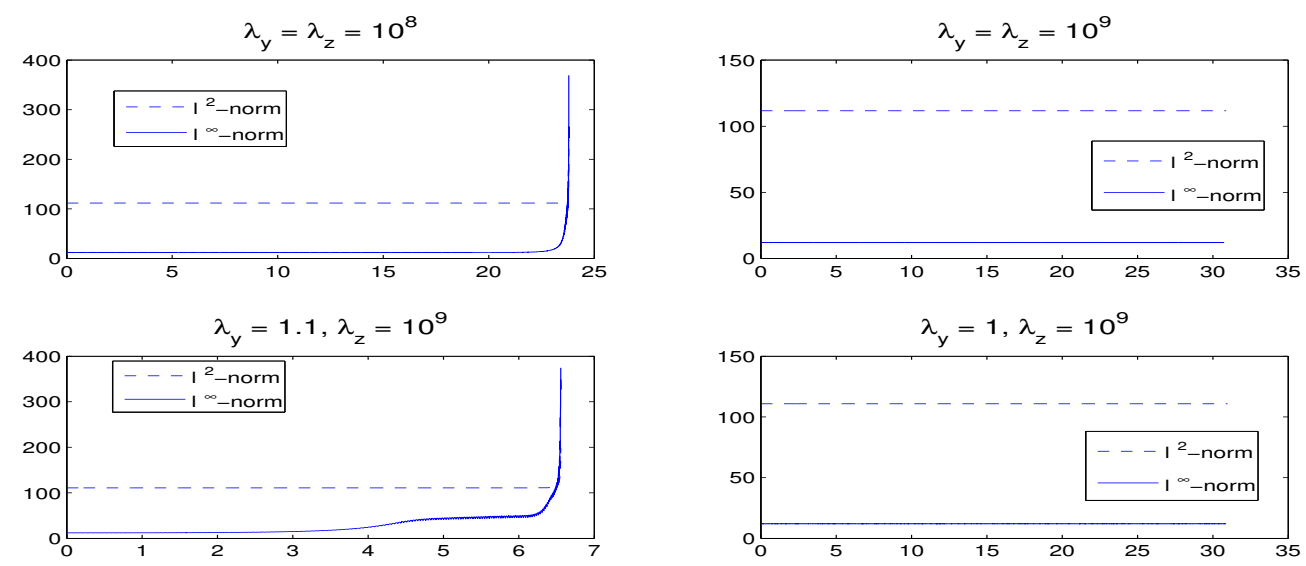

Figure 31: Evolutions of the $l^{2}$-norm (---) and the $l^{\infty}$-norm (-) with respect to time, for $p=1, a=b=-1, c=4, K=32, L=M=4, N_{x}=256, N_{y}=N_{z}=16, \Delta t=5 \times 10^{-4}$. Here $\lambda_{y}=10^{9}, \lambda_{z}=50,10^{9}$ and $\varepsilon=5 \times 10^{-3}$.

Figures 32 - 34 show the results obtained in the contexts where $a b=-1$, with both small and large values of wavelengths. Let us mention that the same results as in Figure 32 are obtained from simulations when $a=1, b=-1$. The behaviors of the norms differ when $\lambda_{y} \neq \lambda_{z}$ and in the contexts where $a b=-1$.
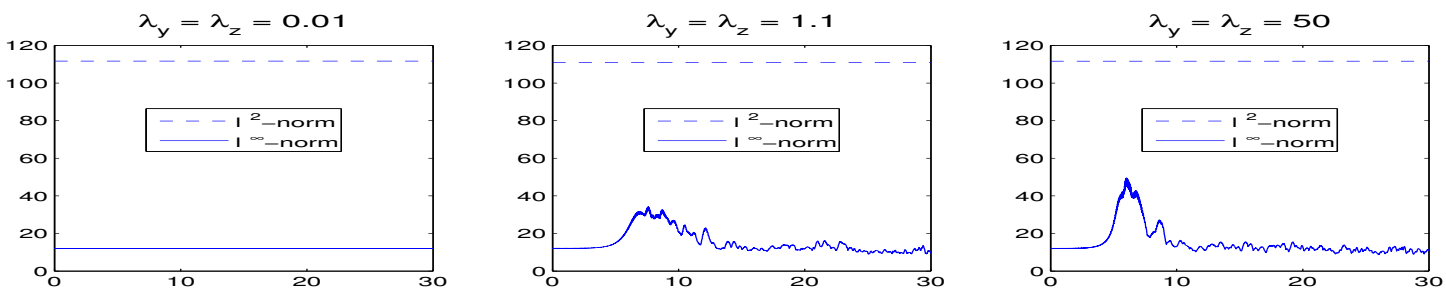

Figure 32: Evolutions of the $l^{2}$-norm (--) and the $l^{\infty}$-norm (-) with respect to time, for $p=1, a=-1, b=1, c=4, K=32, L=M=4, N_{x}=256, N_{y}=N_{z}=16, \Delta t=5 \times 10^{-4}$. Here, $\lambda_{y}=\lambda_{z}=0.01,1.1,50$ and $\varepsilon=5 \times 10^{-3}$.

For this smaller value of $\varepsilon$, we report in fact the same observations as before. In particular, for $a=b=-1$, there exist two critical transverse wavelengths $\lambda_{4}^{1} \simeq 1.1$ and $\lambda_{4}^{2} \gg \lambda_{4}^{1}$, such that if $\lambda_{4}^{1}<\lambda_{y}<\lambda_{4}^{2}$ or $\lambda_{4}^{1}<\lambda_{z}<\lambda_{4}^{2}$, the perturbed line-soliton blows up. 

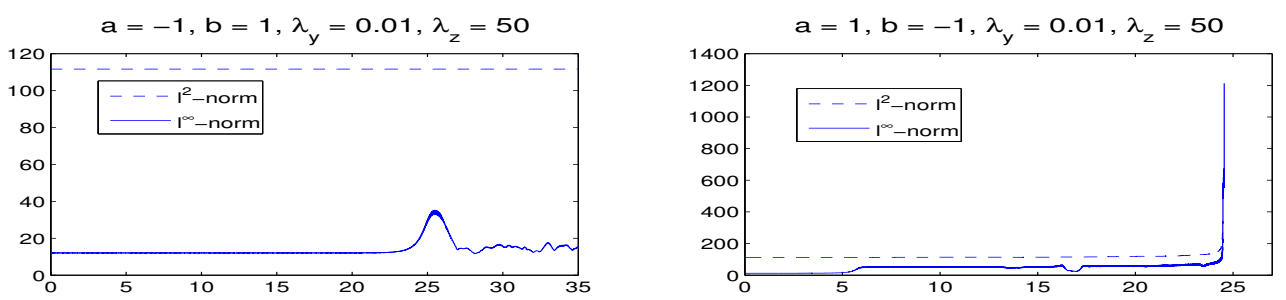

Figure 33: Evolutions of the $l^{2}-$ norm (--) and the $l^{\infty}$-norm (-) with respect to time, for $p=1, a b=-1, c=4, K=32, L=M=4, N_{x}=256, N_{y}=N_{z}=16, \Delta t=5 \times 10^{-4}$. Here, $\lambda_{y}=0.01, \lambda_{z}=50$ and $\varepsilon=5 \times 10^{-3}$.
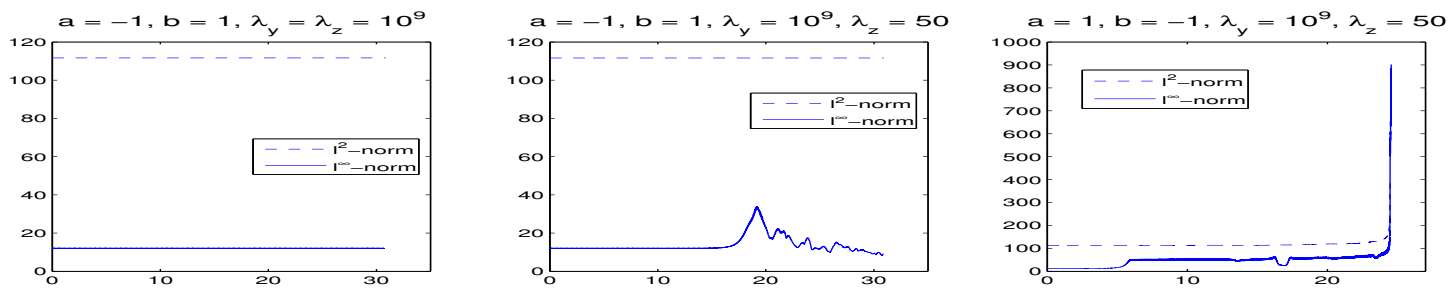

Figure 34: Evolutions of the $l^{2}-$ norm (--) and the $l^{\infty}$-norm (-) with respect to time, for $p=1, a b=-1, c=4, K=32, L=M=4, N_{x}=256, N_{y}=N_{z}=16, \Delta t=5 \times 10^{-4}$. Here $\lambda_{y}=10^{9}, \lambda_{z}=50,10^{9}$ and $\varepsilon=5 \times 10^{-3}$.

\subsubsection{Periodicity in time of the surface}

When the wavelengths $\lambda_{y}$ and $\lambda_{z}$ do not satisfy the resulting "instability condition", i.e. when $c \lambda_{y}, c \lambda_{z}<4.2$, a periodicity in time of the perturbed line-soliton's oscillations is observed. Indeed, if we consider a reference frame moving at the velocity $c$, the amplitude of the top wave, $\sup _{x} u(x, y, z, 0)$, is given by

$$
A_{c}(y, z)=3 c\left(1+\varepsilon \cos \left(\frac{2 \pi y}{\lambda_{y}}+\frac{2 \pi z}{\lambda_{z}}\right)\right),
$$

and represents the oscillations of the perturbed line-soliton's surface around the value $3 c$. We notice that the maximal amplitude of the perturbed line-soliton is reached for $y=z=0$, and is equal to $3 c$.

We fix in this subsection $\varepsilon=10^{-2}, K=32, L=M=4, N_{x}=256, N_{y}=N_{z}=16$, $\Delta t=5 \times 10^{-4}$. In Figure 35, we represent, when $a=b=-1$, the evolution of $\sup _{x} u(x, 0,0, t)$, where $u$ is the discrete solution obtained respectively with $c=2, \lambda_{y}=\lambda_{z}=1,2$, and $c=4$, $\lambda_{y}=\lambda_{z}=1$. 

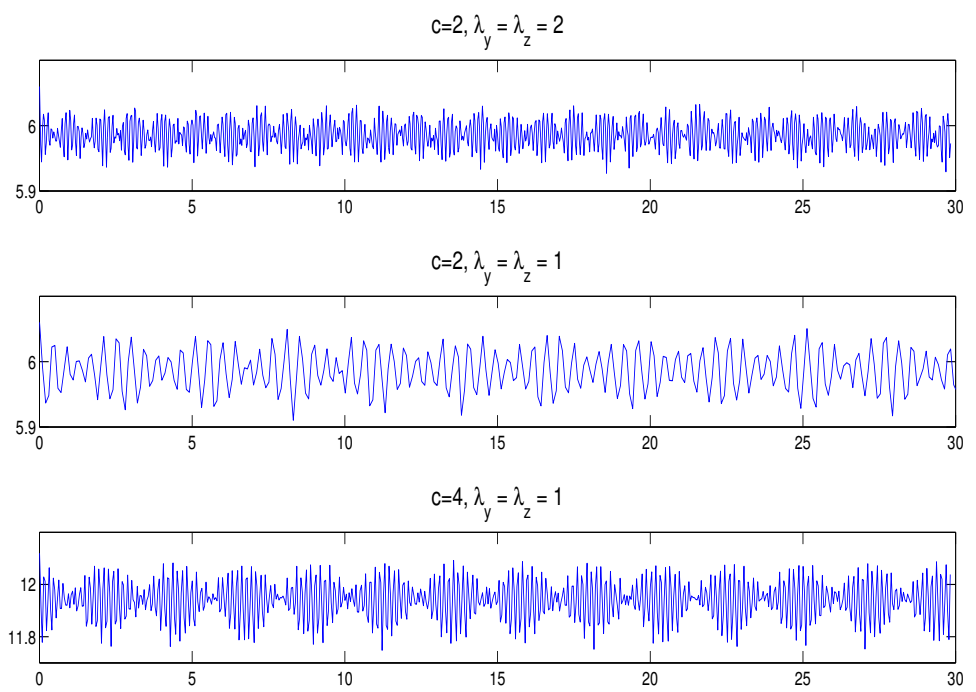

Figure 35: Evolution of $\sup _{x} u(x, 0,0, t)$ with respect to time, for $p=1, a=b=-1, c=2,4, K=$ $32, L=M=4, N_{x}=256, N_{y}=N_{z}=16, \Delta t=5 \times 10^{-4}$. Here, $\lambda_{y}=\lambda_{z}=2,1$ and $\varepsilon=10^{-2}$.

For each fixed value of $c$, the period increases when $\lambda_{y}$ and $\lambda_{z}$ decrease. Also, for fixed values of $\lambda_{y}, \lambda_{z}$, the period increases when $c$ decreases.

Another form of stability appears in the contexts where $a b=-1$. The experiments associated with the results represented in Figure 36 consider $a=1, b=-1$ and $\lambda_{y}=\lambda_{z}=50$.
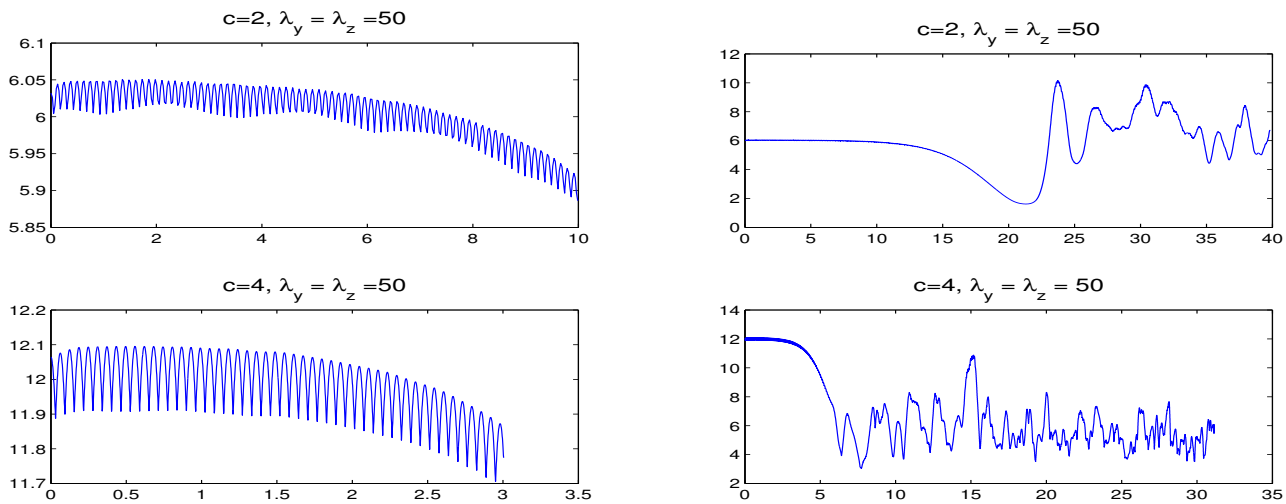

Figure 36: Evolution of $\sup _{x} u(x, 0,0, t)$ with respect to time, for $p=1, a=1, b=-1, c=$ $2,4, K=32, L=M=4, N_{x}=256, N_{y}=N_{z}=16, \Delta t=5 \times 10^{-4}$. Here, $\lambda_{y}=\lambda_{z}=50$ and $\varepsilon=10^{-2}$. 
When $c=4$, the periodicity is lost at the instant $T \simeq 3$. If we compare with the results of Figure 26, we notice in fact that from this instant, the solution has a tendency to blow up.

\subsection{Generalized case: $p=2$}

We want here to study the transverse instabilities in the case where $p=2$.

\subsubsection{Evolution in long time}

Our experiments are performed in each of the contexts where $a b= \pm 1$, by fixing $K=32$, $L=M=4, N_{x}=256, N_{y}=N_{z}=16$ and $\Delta t=5 \times 10^{-4}$. We start by considering $\varepsilon=10^{-2}$, and the context where $a=b=-1$. Figure 37 presents, for $c=1$, the evolutions of the $l^{2}$ and $l^{\infty}$-norms of the discrete solution obtained respectively with $\lambda_{y}=\lambda_{z}=0.01,0.5,0.6$ and 50 .
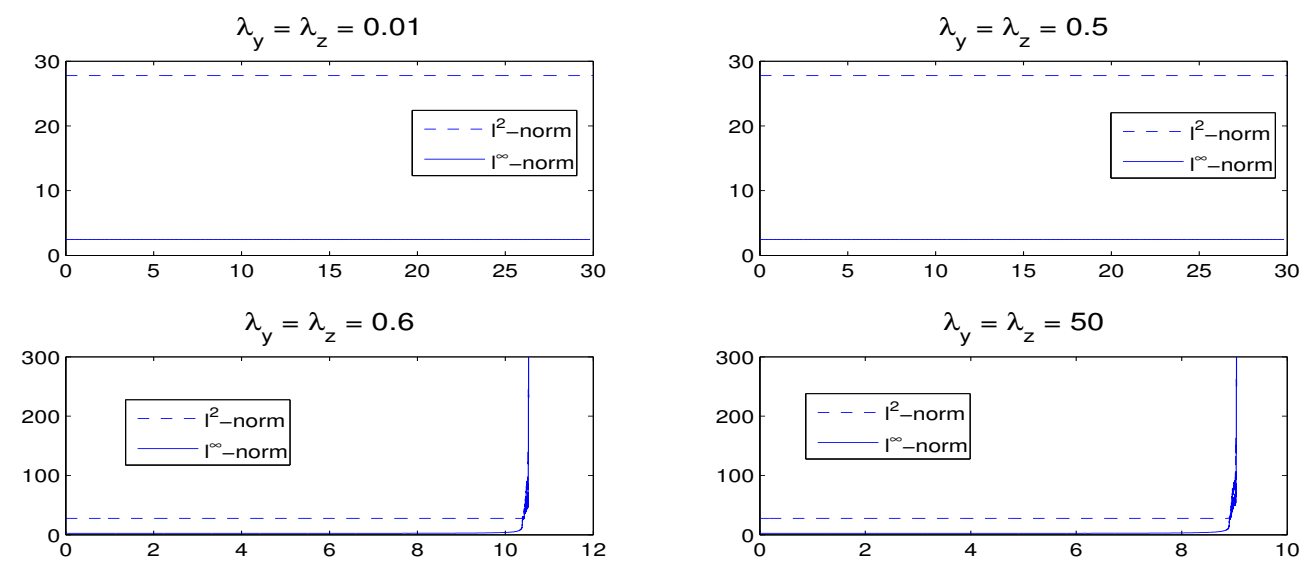

Figure 37: Evolutions of the $l^{2}-$ norm (--) and the $l^{\infty}$-norm (-) with respect to time, for $p=2, a=b=-1, c=1, K=32, L=M=4, N_{x}=256, N_{y}=N_{z}=16, \Delta t=5 \times 10^{-4}$. Here, $\lambda_{y}=\lambda_{z}=0.01,0.5,0.6,50$ and $\varepsilon=10^{-2}$.

As Figure 38 shows, similar results derive from simulations with a smaller value of $\varepsilon$, namely with $\varepsilon=5 \times 10^{-3}$. 

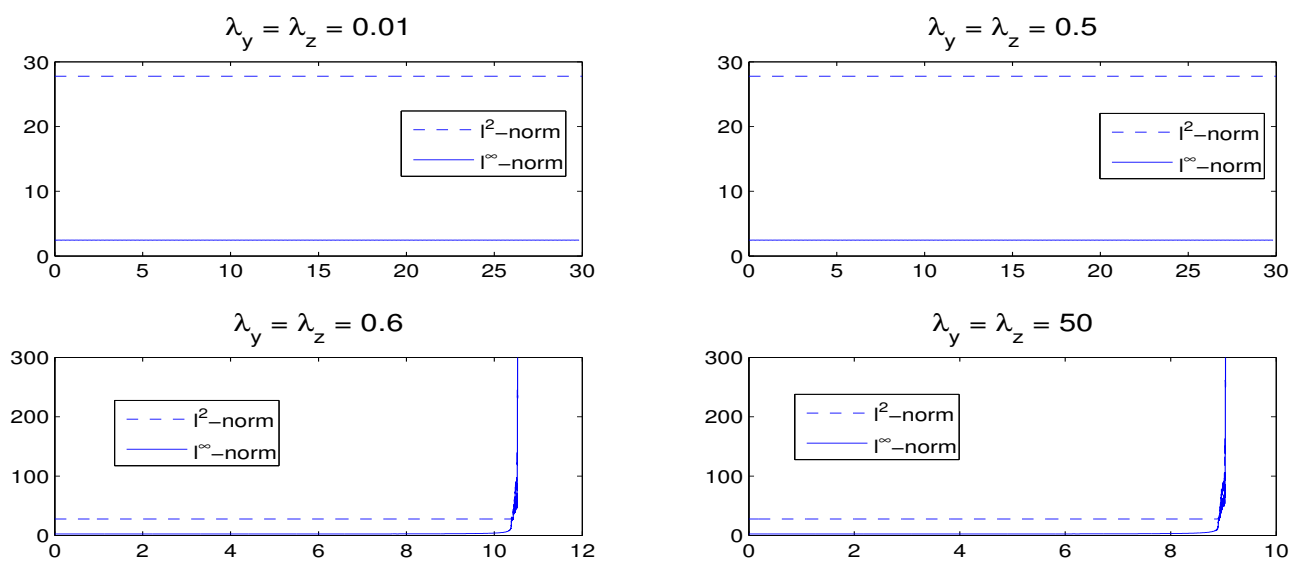

Figure 38: Evolutions of the $l^{2}$-norm (---) and the $l^{\infty}$-norm (-) with respect to time, for $p=2, a=b=-1, c=1, K=32, L=M=4, N_{x}=256, N_{y}=N_{z}=16, \Delta t=5 \times 10^{-4}$. Here, $\lambda_{y}=\lambda_{z}=0.01,0.5,0.6,50$ and $\varepsilon=5 \times 10^{-3}$.

Independently of these small values of $\varepsilon$, we notice that there exists once again a critical transverse wavelength $\lambda_{1}^{1} \simeq 0.6$ such that if $\lambda_{y}$ and $\lambda_{z}$ are larger than $\lambda_{1}^{1}$, the perturbed line-soliton blows up.

Figure 39 shows the results obtained, again for $\varepsilon=10^{-2}$ and $c=1$, but now from distinct wavelengths; $\lambda_{y}=0.01,0.5$, and $\lambda_{z}=0.6,50$.
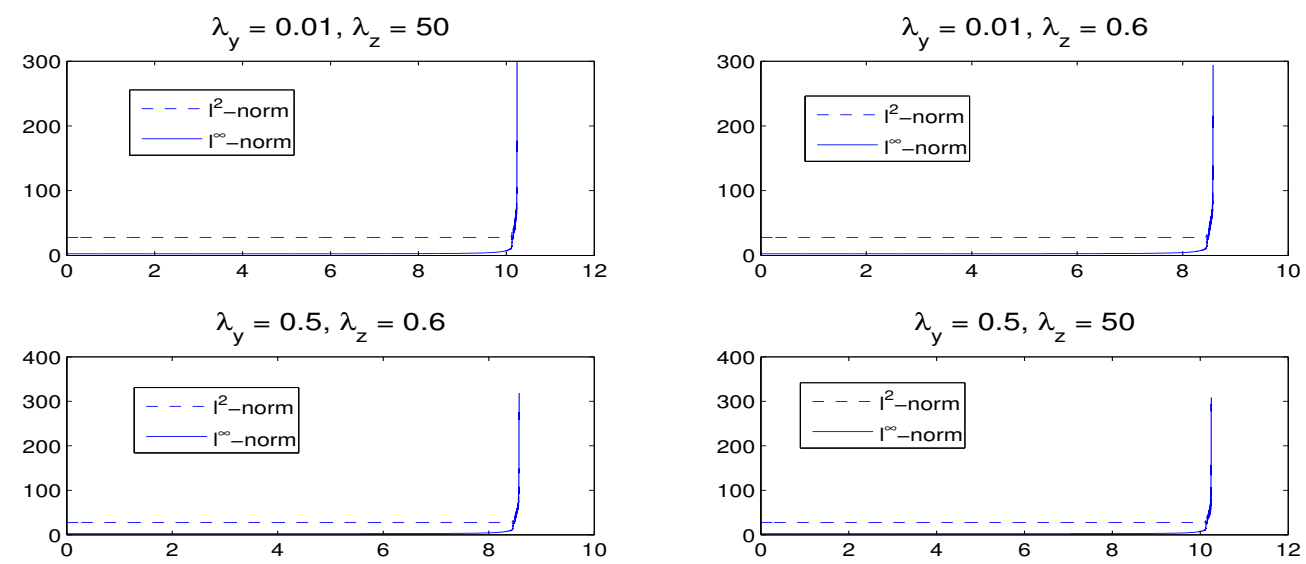

Figure 39: Evolutions of the $l^{2}$-norm (--) and the $l^{\infty}$-norm (-) with respect to time, for $p=2, a=b=-1, c=1, K=32, L=M=4, N_{x}=256, N_{y}=N_{z}=16, \Delta t=5 \times 10^{-4}$. Here, $\lambda_{y}=0.01,0.5, \lambda_{z}=0.6,50$ and $\varepsilon=10^{-2}$. 
As it was the case for $p=1$, there exists a critical transverse wavelength $\lambda_{1}^{1} \simeq 0.6$ such that if $\lambda_{y}$ or $\lambda_{z}$ is larger than $\lambda_{1}^{1}$, the perturbed line-soliton blows up.

Let us now inspect the influence of the velocity as regards these observations. The change of functions

$$
\widetilde{u}(x, y, z, t):=\frac{1}{c^{1 / p}} u\left(\frac{x}{\sqrt{c}}, \frac{y}{c}, \frac{z}{c}, \frac{t}{c \sqrt{c}}\right),
$$

applied to (2), gives

$$
\widetilde{u}_{t}+\widetilde{u}^{p} \widetilde{u}_{x}+\widetilde{u}_{x x x}+a \partial_{x}^{-1} \widetilde{u}_{y y}+b \partial_{x}^{-1} \widetilde{u}_{z z}=0 .
$$

The initial datum becomes then

$\widetilde{u}_{0}(x, y, z)=\left(\frac{(p+1)(p+2)}{2}\right)^{1 / p}\left(1+\varepsilon \cos \left(\frac{2 \pi y}{c \lambda_{y}}+\frac{2 \pi z}{c \lambda_{z}}\right)\right)^{1 / p} \operatorname{sech}\left(\frac{p x}{2} \sqrt{1+\varepsilon \cos \left(\frac{2 \pi y}{c \lambda_{y}}+\frac{2 \pi z}{c \lambda_{z}}\right)}\right)$.

We notice that propagating a line-soliton of velocity $c$, perturbed in the two transverse directions, is equivalent to propagating a line-soliton of velocity equal to 1 perturbed in the same directions by the wavelengths $c \lambda_{y}$ and $c \lambda_{z}$.

The experiments, with $c=1$, show that there exists a critical transverse wavelength $\lambda_{1}^{1} \simeq 0.6$. The previous change of functions indicates that if $c \lambda_{y}$ or $c \lambda_{z}$ is larger than $\lambda_{1}^{1}$, then the perturbed line-soliton is unstable.

Let us confirm this indication by taking $c=2$. The results represented in Figure 40 are obtained for equal wavelengths: $\lambda_{y}=\lambda_{z}=0.01,0.2,0.3$ and 50 .
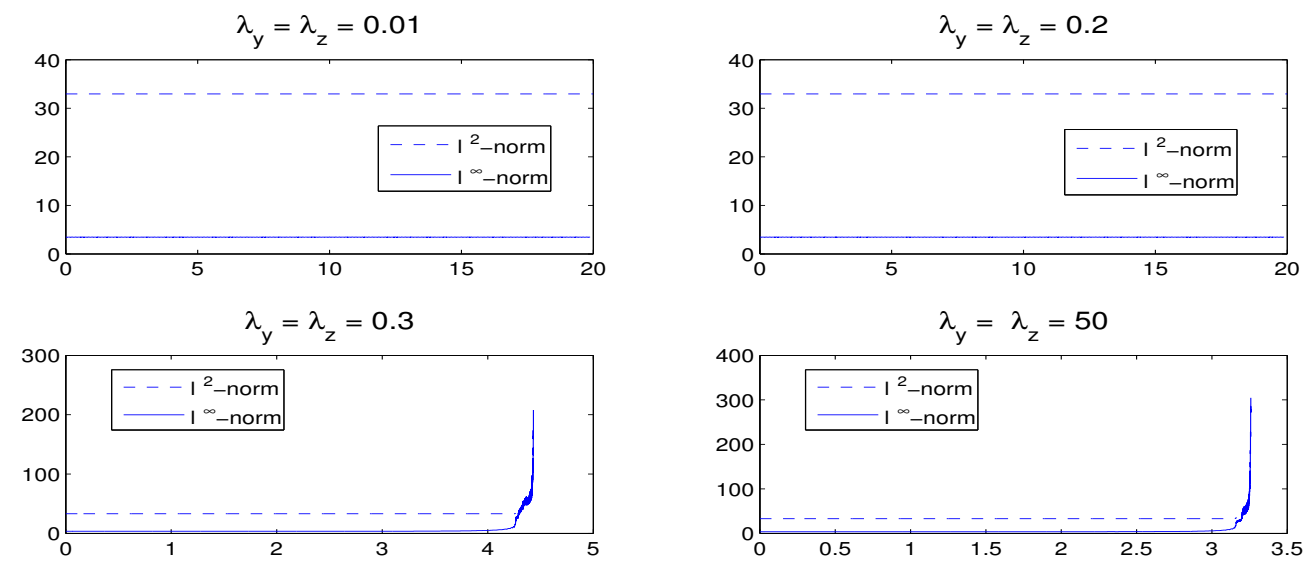

Figure 40: Evolutions of the $l^{2}$-norm (--) and the $l^{\infty}$-norm (-) with respect to time, for $p=2, a=b=-1, c=2, K=32, L=M=4, N_{x}=256, N_{y}=N_{z}=16, \Delta t=5 \times 10^{-4}$. Here, $\lambda_{y}=\lambda_{z}=0.01,0.2,0.3,50$ and $\varepsilon=10^{-2}$. 
By reproducing the previous experiments but with distinct wavelengths, $\lambda_{y}=0.01,0.2$, $\lambda_{z}=0.3,50$, we obtain the results represented in Figure 41.
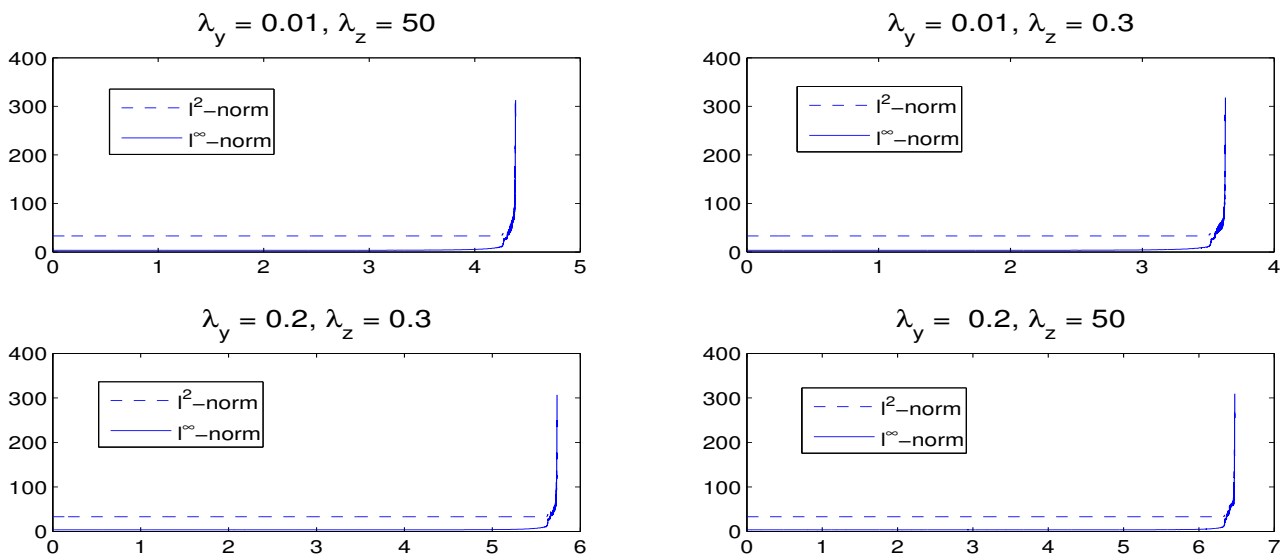

Figure 41: Evolutions of the $l^{2}-$ norm (--) and the $l^{\infty}$-norm (-) with respect to time, for $p=2, a=b=-1, c=2, K=32, L=M=4, N_{x}=256, N_{y}=N_{z}=16, \Delta t=5 \times 10^{-4}$. Here, $\lambda_{y}=0.01,0.2, \lambda_{z}=0.3,50$ and $\varepsilon=10^{-2}$.

In what follows, we perform experiments in the contexts where $a b=-1$, and choose to display the results only for a velocity $c=2$; the observations for $c=1$ being similar. Figure 42 presents in the situation where $a=-1, b=1$, the evolutions of the $l^{2}$ - and $l^{\infty}$-norms of the discrete solution obtained respectively for $\lambda_{y}=\lambda_{z}=0.01,0.2,0.3$ and 50 .
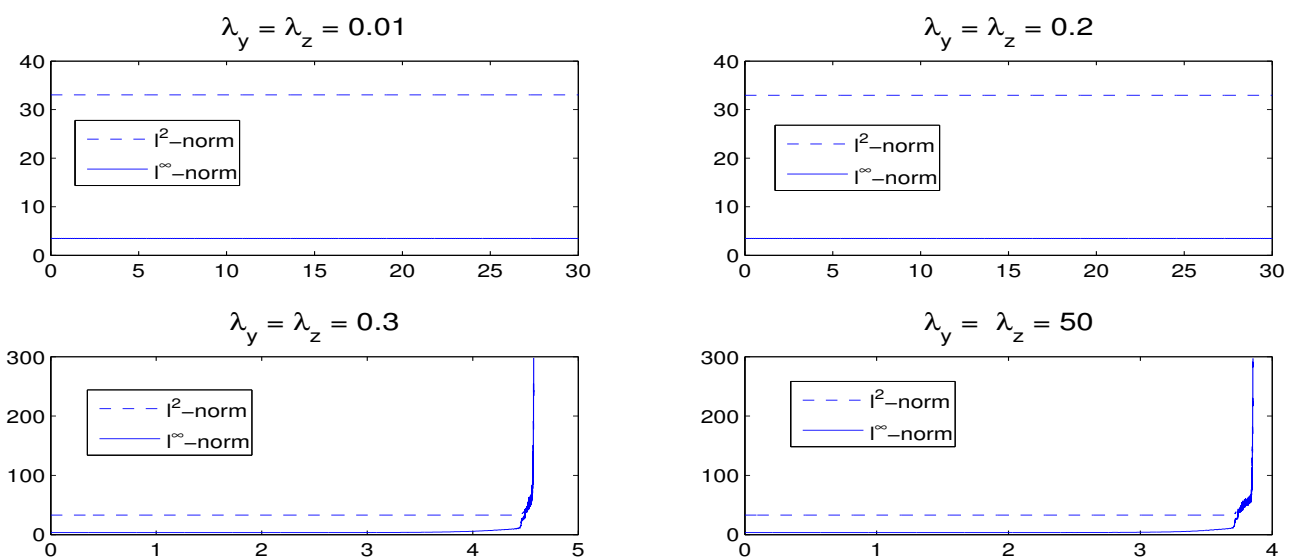

Figure 42: Evolutions of the $L^{2}$-norm (--) and the $L^{\infty}$-norm $(-)$ with respect to time, for $p=2, a=-1, b=1, c=2, K=32, L=M=4, N_{x}=256, N_{y}=N_{z}=16, \Delta t=5 \times 10^{-4}$. Here, $\lambda_{y}=\lambda_{z}=0.01,0.2,0.3,50$ and $\varepsilon=10^{-2}$. 
The same evolutions of the norms derive from the simulations when we consider $a=1$, $b=-1$; this is justified from the argument previously mentioned in the context where $\lambda_{y}=\lambda_{z}$.

Figure 43 presents the results obtained by reproducing the experiments in the context where $a=-1, b=1$, but for distinct wavelengths; $\lambda_{y}=0.01,0.2$ and $\lambda_{z}=0.3,50$.
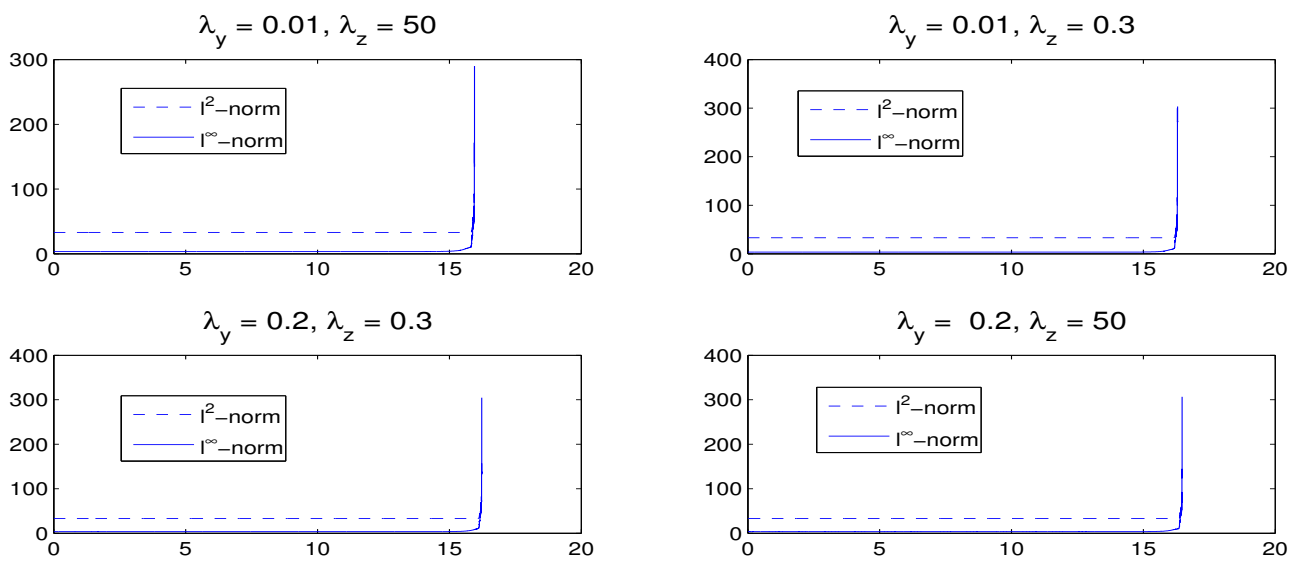

Figure 43: Evolutions of the $l^{2}$-norm (---) and the $l^{\infty}$-norm (-) with respect to time, for $p=2, a=-1, b=1, c=2, K=32, L=M=4, N_{x}=256, N_{y}=N_{z}=16, \Delta t=5 \times 10^{-4}$. Here, $\lambda_{y}=0.01,0.2, \lambda_{z}=0.3,50$ and $\varepsilon=10^{-2}$.

By considering now the context where $a=1, b=-1$, with the previous distinct wavelengths, we obtain the results represented in Figure 44.
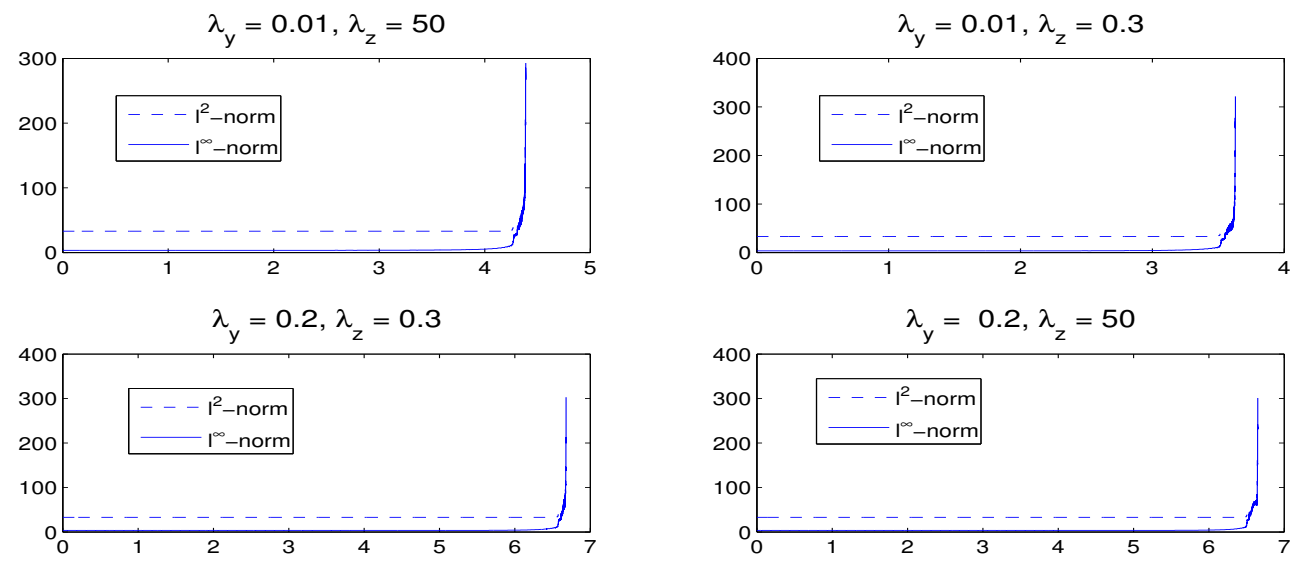

Figure 44: Evolutions of the $l^{2}$-norm (--) and the $l^{\infty}$-norm (-) with respect to time, for $p=2, a=1, b=-1, c=2, K=32, L=M=4, N_{x}=256, N_{y}=N_{z}=16, \Delta t=5 \times 10^{-4}$. Here, $\lambda_{y}=0.01,0.2, \lambda_{z}=0.3,50$ and $\varepsilon=10^{-2}$. 
As in the situation where $a=b=-1$, there exists also here a critical transverse wavelength, $\lambda_{2}^{1} \simeq 0.3$, such that if $\lambda_{y}$ or $\lambda_{z}$ is larger than $\lambda_{2}^{1}$, then the perturbed line-soliton is unstable.

Let us now inspect numerically the following phenomenon studied in [2]. Of course, when $\lambda_{y}$ and $\lambda_{z}$ tend to infinity, the formal limit of the perturbed line-soliton gives $\Phi_{c(1+\varepsilon)}$, which is independent of the transverse variables; the solution of (2) with $\Phi_{c(1+\varepsilon)}$ as initial datum is stable as long as $p<4$ (see [2]). In the experiments associated with Figure 45, we have considered $a=b=-1$ and used very large wavelengths; $\lambda_{y}=0.2,50,10^{8}, 10^{9}$ and $\lambda_{z}=10^{8}, 10^{9}$.
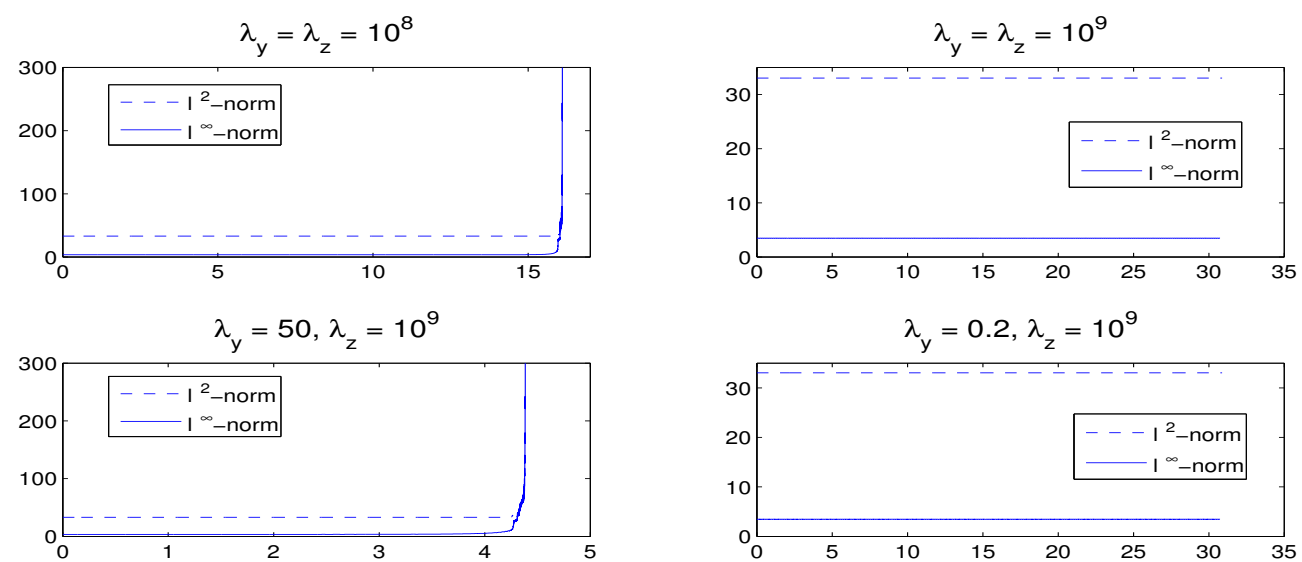

Figure 45: Evolutions of the $l^{2}-$ norm (--) and the $l^{\infty}$-norm (-) with respect to time, for $p=2, a=b=-1, c=2, K=32, L=M=4, N_{x}=256, N_{y}=N_{z}=16, \Delta t=5 \times 10^{-4}$. Here, $\lambda_{y}=0.2,50,10^{8}, 10^{9}, \lambda_{z}=10^{8}, 10^{9}$ and $\varepsilon=10^{-2}$.

There exists another critical transverse wavelength $\lambda_{c}^{2}$ such that if $\lambda_{y}, \lambda_{z}>\lambda_{c}^{2}$, the perturbed line-soliton no longer blows up. The same observation derives from simulations when $a b=-1$.

In the last context where $a=b=1$, Figure 46 shows the results obtained with both equal and distinct wavelengths.

The perturbed line-soliton seems to never blow up; this observation also results from simulations when we consider $c=1$, or use larger values for $K, L, M, N_{x}, N_{y}, N_{z}$ (e.g. with $K=50, L=M=$ $\left.6, N_{x}=512, N_{y}=N_{z}=32\right)$. 

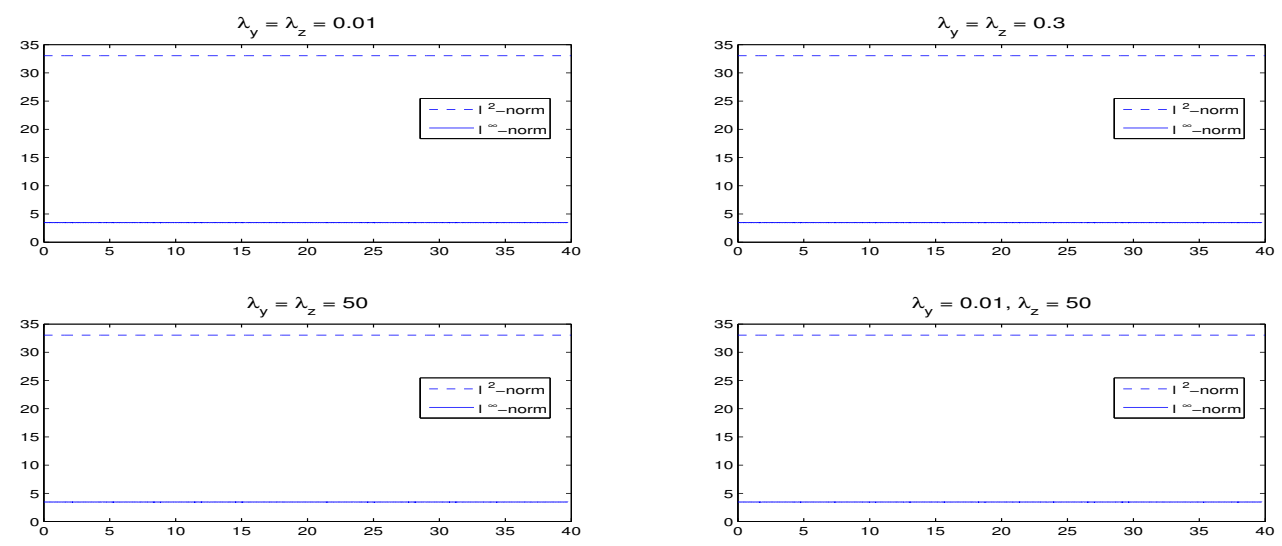

Figure 46: Evolutions of the $l^{2}$-norm (--) and the $l^{\infty}$-norm $(-)$ with respect to time for $p=2, a=b=1, c=2, K=32, L=M=4, N_{x}=256, N_{y}=N_{z}=16, \Delta t=5 \times 10^{-4}$. Here, $\lambda_{y}=\lambda_{z}=0.01,0.3,50, \lambda_{y}=0.01, \lambda_{z}=50$ and $\varepsilon=10^{-2}$.

\subsubsection{Periodicity in time of the surface}

As it was the case for $p=1$, when the wavelengths $\lambda_{y}$ and $\lambda_{z}$ do not satisfy the resulting "instability condition", i.e. when $c \lambda_{y}, c \lambda_{z}<0.6$, a periodicity in time of the perturbed linesoliton's oscillations is observed. In fact, in a reference frame moving at the velocity $c$, the amplitude of the top wave, $\sup _{x} u(x, y, z, 0)$, is given by

$$
A_{c}(y, z)=\left(\frac{(p+1)(p+2) c}{2}\right)^{1 / p}\left(1+\varepsilon \cos \left(\frac{2 \pi y}{\lambda_{y}}+\frac{2 \pi z}{\lambda_{z}}\right)\right)^{1 / p}
$$

Since $\varepsilon$ is sufficiently small, we have $A_{c}(y, z) \simeq\left(\frac{(p+1)(p+2) c}{2}\right)^{1 / p}\left(1+\frac{\varepsilon}{p} \cos \left(\frac{2 \pi y}{\lambda_{y}}+\frac{2 \pi z}{\lambda_{z}}\right)\right)$ representing the oscillations of the perturbed line-soliton's surface around the value $\left(\frac{(p+1)(p+2) c}{2}\right)^{1 / p}$. The maximal amplitude of the perturbed line-soliton is reached for $y=z=0$, and is equal to $\left(\frac{(p+1)(p+2) c}{2}\right)^{1 / p}$.

Let us fix here $a=b=-1, \varepsilon=10^{-2}, K=32, L=M=4, N_{x}=256, N_{y}=N_{z}=16$ and $\Delta t=5 \times 10^{-4}$. We represent in Figure 47 the evolution of $\sup _{x} u(x, 0,0, t)$, where $u$ is the discrete solution obtained respectively with $c=1, \lambda_{y}=\lambda_{z}=0.2,0.5$, and $c=2, \lambda_{y}=\lambda_{z}=0.2$.

We observe that, for $c$ fixed, the period increases when $\lambda_{y}$ and $\lambda_{z}$ decrease. Also for fixed values of $\lambda_{y}, \lambda_{z}$, the period increases when $c$ decreases.

Let us now summarize the numerical observations of this section. The experiments for $p=1,2$ show that when the line-soliton is perturbed in the two transverse directions by neither 

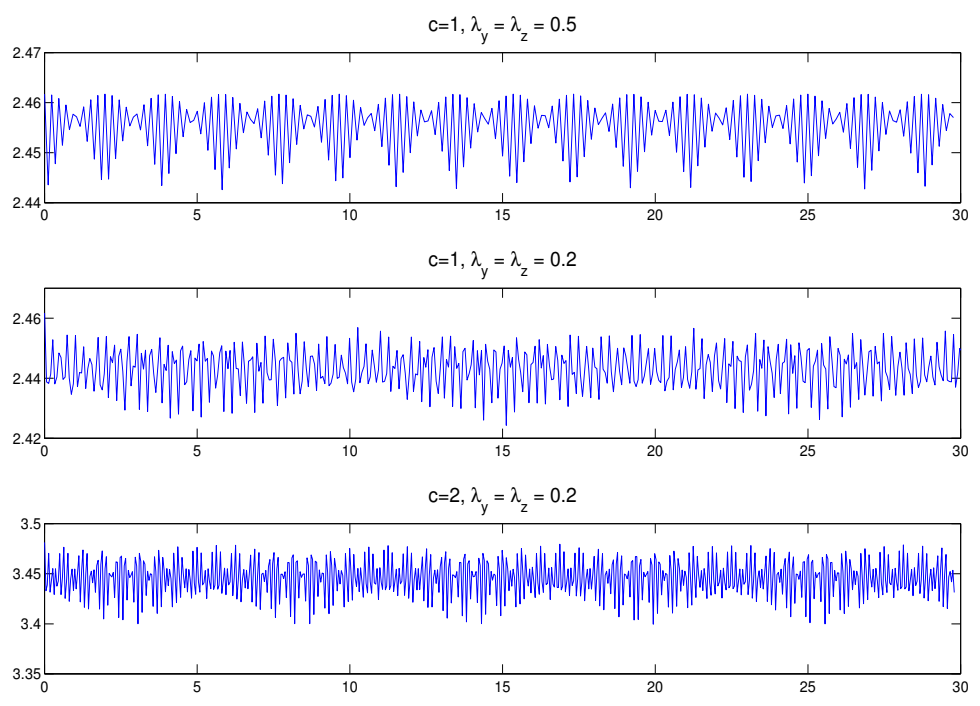

Figure 47: Evolution of $\sup _{x} u(x, 0,0, t)$ with respect to time, for $p=2, a=b=-1, c=$ $1,2, K=32, L=M=4, N_{x}=256, N_{y}=N_{z}=16, \Delta t=5 \times 10^{-4}$. Here, $\lambda_{y}=\lambda_{z}=0.5,0.2$ and $\varepsilon=10^{-2}$.

very small nor very large wavelengths, the associated discrete solution blows up. More precisely, when $p=1,2$ and $a=b=-1$, respectively when $p=2$ and $a b=-1$, we observe that there exist two critical transverse wavelengths $0<\lambda_{c}^{1} \ll \lambda_{c}^{2}$, depending only on $c$, such that the discrete solution, with the perturbed line-soliton as initial datum, blows up (and is hence unstable) if $\lambda_{c}^{1}<\lambda_{y}<\lambda_{c}^{2}$ or $\lambda_{c}^{1}<\lambda_{z}<\lambda_{c}^{2}$. Typically, $\lambda_{c}^{1} \simeq \frac{4.2}{c}$ when $p=1$, and $\lambda_{c}^{1} \simeq \frac{0.6}{c}$ when $p=2$. Let us mention that, in the (2D- ) context of the generalized KP-I equation (with $p=2$ ), a similar numerical study achieved in [9] provides a critical wavelength approximately equal to $\frac{5.559}{c}$.

Another interesting observation is the one reported when $p=1$ and $a=1, b=-1$, respectively $a=-1, b=1$. We notice that the discrete solution, with the perturbed line-soliton as initial datum, blows up if $\lambda_{c}^{1}<\lambda_{z}<\lambda_{c}^{2}$, respectively $\lambda_{c}^{1}<\lambda_{y}<\lambda_{c}^{2}$, and if the wavelength in the positive transverse direction is either small $\left(\lambda_{y}<\lambda_{c}^{1}\right.$, respectively $\left.\lambda_{z}<\lambda_{c}^{1}\right)$ or very large $\left(\lambda_{y}>\lambda_{c}^{2}\right.$, respectively $\left.\lambda_{z}>\lambda_{c}^{2}\right)$.

These observations lead us to formulate the following conjecture.

\section{Conjecture 4.1}

For $\varepsilon>0$ sufficiently small, let $u_{0}$ be the perturbed line-soliton of velocity $c(y, z):=c(1+$ $\left.\varepsilon \cos \left(\frac{2 \pi y}{\lambda_{y}}+\frac{2 \pi z}{\lambda_{z}}\right)\right)$, where $\lambda_{y}, \lambda_{z}>0$.

Let $p \geq 1$, and $a=b=-1$. There exist two critical transverse wavelengths $0<\lambda_{c}^{1} \ll \lambda_{c}^{2}$, 
depending only on $c$, such that the solution of the generalized 3D-KP equation (2), with $u_{0}$ as initial datum, blows up in finite time (in particular the line-soliton is unstable under the flow of (2)) if $\lambda_{c}^{1}<\lambda_{y}<\lambda_{c}^{2}$ or $\lambda_{c}^{1}<\lambda_{z}<\lambda_{c}^{2}$.

Let $p \geq 2$, and $a=1, b=-1$, respectively $a=-1, b=1$. There exist two critical transverse wavelengths $0<\lambda_{c}^{1} \ll \lambda_{c}^{2}$, depending only on $c$, such that the solution of the generalized $3 D-K P$ equation (2), with $u_{0}$ as initial datum, blows up in finite time if $\lambda_{c}^{1}<\lambda_{y}<\lambda_{c}^{2}$ or $\lambda_{c}^{1}<\lambda_{z}<\lambda_{c}^{2}$.

Let $p=1$, and $a=1, b=-1$, respectively $a=-1, b=1$. There exist two critical transverse wavelengths $0<\lambda_{c}^{1} \ll \lambda_{c}^{2}$, depending only on $c$, such that the solution of the generalized $3 D-K P$ equation (2), with $u_{0}$ as initial datum, blows up in finite time if $\lambda_{c}^{1}<\lambda_{z}<\lambda_{c}^{2}, \lambda_{y}<\lambda_{c}^{1}$ or $\lambda_{y}>\lambda_{c}^{2}$, respectively $\lambda_{c}^{1}<\lambda_{y}<\lambda_{c}^{2}, \lambda_{z}<\lambda_{c}^{1}$ or $\lambda_{z}>\lambda_{c}^{2}$.

\section{Transverse instability in the $z$-direction}

We are concerned in this section with the perturbation of the profiles of the Zaitsev [24] traveling waves. The function

$$
\psi_{c}(x, y, t)=12 \alpha^{2} \frac{1-\beta \cosh (\alpha x-\omega t) \cos (\delta y)}{(\cosh (\alpha x-\omega t)-\beta \cos (\delta y))^{2}}
$$

with

$$
\beta=\sqrt{\frac{\delta^{2}-3 \alpha^{4}}{\delta^{2}}}, 3 \alpha^{4}<\delta^{2}, \omega=\frac{\delta^{2}+\alpha^{4}}{\alpha}, c=\frac{\omega}{\alpha},
$$

which is localized in the $x$-direction and periodic in the $y$-direction, represents such a profile, and satisfies KP-I (equation (1) with $a=-1$ ). As mentioned earlier, in the situation where $\beta$ tends to 1 , with $\delta$ and $\alpha$ close to $0, \psi_{c}$ corresponds to the solution of KP-I so called the lump-soliton. Here, we will associate with $\delta$ and $\alpha$ small values, and improperly call $\psi_{c}(x, y, 0)$ the "Zaitsev wave".

We want to study the evolution, subject to (2), of the Zaitsev wave when it is perturbed in the second transverse direction. We then consider

$$
u_{0}(x, y, z):=\psi_{c(z)}(x, y, 0)
$$

as the initial datum associated with (2). The velocity is here slightly perturbed in the $z$-direction; namely, $c(z):=c\left(1+\varepsilon \cos \left(\frac{2 \pi z}{\lambda_{z}}\right)\right)$, with $\varepsilon>0$ sufficiently small and $\lambda_{z}>0$ a wavelength.

We fix in this section $p=1$, and $a=-1$. Our numerical simulations will be performed in each of the contexts where $b= \pm 1$. 


\subsection{Evolution in long time}

In what follows, we consider $K=32, L=12, M=4, N_{x}=256, N_{y}=32, N_{z}=16$, $\Delta t=5 \times 10^{-4}$. The value of $L$ is larger than in the previous sections in such a way that the present initial datum $u_{0}$ also satisfies (5) numerically. Moreover, this value allows us to consider $\delta=\frac{\pi}{L}$ in such a way that we can affect to $\alpha$ values that are not very small (recalling that $3 \alpha^{4}<\delta^{2}$ ). In the presentation of the results of this section, we will only specify the values of $c$ deriving then from such choices of $\delta$ and $\alpha$. Let us set on the other hand $\varepsilon=10^{-2}$. The results represented in Figure 48 concern the context where $b=-1$, and show for $c=1$ the evolutions of the $l^{2}$ and $l^{\infty}$-norms of the discrete solution obtained respectively with $\lambda_{z}=0.01$, 0.5, 0.6 and 50. Similar results derive from simulations, even by using a slightly bigger time step, e.g. $\Delta t=10^{-3}$, with larger values of $K, L, N_{x}, N_{y}$ (e.g. with $K=50, L=24, N_{x}=512, N_{y}=64$ ).
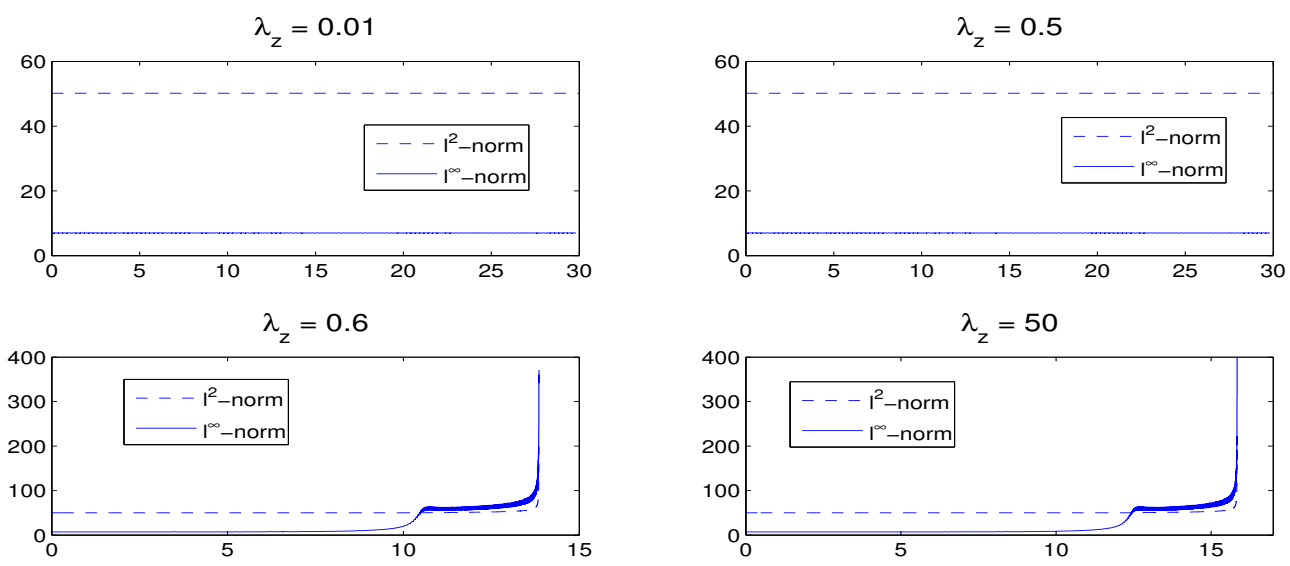

Figure 48: Evolutions of the $l^{2}-$ norm $(--)$ and the $l^{\infty}$-norm (-) with respect to time, for $b=-1, c=1, K=32, L=12, M=4, N_{x}=256, N_{y}=32, N_{z}=16, \Delta t=5 \times 10^{-4}$. Here, $\lambda_{z}=0.01,0.5,0.6,50$ and $\varepsilon=10^{-2}$. 
We notice that there exists a critical transverse wavelength $\lambda_{1}^{1} \simeq 0.6$ such that if $\lambda_{z}>\lambda_{1}^{1}$, the solution blows up. About the results of this section also, let us already mention that in presence of the blow-up of the $l^{\infty}$-norm, a deterioration of the $l^{2}$-norm, from the blow-up instant, will consequently be observed.

Let us inspect now the influence of the velocity as regards this observation. The change of functions

$$
\widetilde{u}(x, y, z, t):=\frac{1}{c} u\left(\frac{x}{\sqrt{c}}, \frac{y}{c}, \frac{z}{c}, \frac{t}{c \sqrt{c}}\right),
$$

applied to (2), for $p=1$, gives

$$
\widetilde{u}_{t}+\widetilde{u} \widetilde{u}_{x}+\widetilde{u}_{x x x}+a \partial_{x}^{-1} \widetilde{u}_{y y}+b \partial_{x}^{-1} \widetilde{u}_{z z}=0
$$

The initial datum becomes then

$$
\widetilde{u}_{0}(x, y, z)=12 \widetilde{\alpha}^{2} \frac{1-\beta \cosh (\widetilde{\alpha} x) \cos (\widetilde{\delta} y)}{(\cosh (\widetilde{\alpha} x)-\beta \cos (\widetilde{\delta} y))^{2}},
$$

where

$$
\beta=\sqrt{\frac{\widetilde{\delta}^{2}-3 \widetilde{\alpha}^{4}}{\widetilde{\delta}^{2}}}, 3 \widetilde{\alpha}^{4}<\widetilde{\delta}^{2}, \widetilde{\omega}=\frac{\widetilde{\delta}^{2}+\widetilde{\alpha}^{4}}{\widetilde{\alpha}}, \widetilde{c}=\frac{\widetilde{\omega}}{\widetilde{\alpha}}=1 .
$$

The velocity is then perturbed in the $z$-direction: $\widetilde{c}(z)=\left(1+\varepsilon \cos \left(\frac{2 \pi z}{c \lambda}\right)\right)$. We thus notice that propagating a Zaitsev wave of velocity $c$ perturbed in the transverse $z$-direction by the wavelength $\lambda_{z}$ is equivalent to propagating a Zaitsev wave of velocity 1 perturbed in the same direction by the wavelength $c \lambda_{z}$.

The above change of functions indicates that if $\lambda_{z}>\lambda_{c}^{1}:=\frac{\lambda_{1}^{1}}{c}$, the solution blows up in finite time. This is confirmed by the results of Figure 49 obtained also in the context where $b=-1$, but by considering now $c=2$. This figure shows the evolutions of the $l^{2}$ and $l^{\infty}$-norms of the discrete solution, obtained respectively with $\lambda_{z}=0.01,0.2,0.3$ and 50 . 

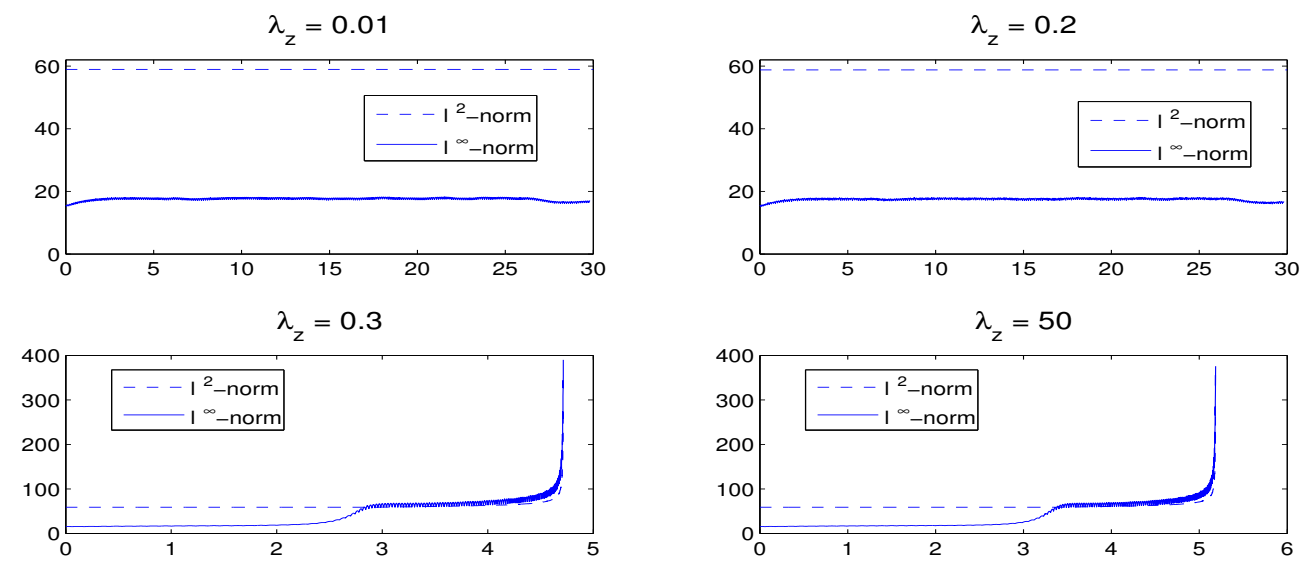

Figure 49: Evolutions of the $l^{2}$-norm (---) and the $l^{\infty}$-norm (-) with respect to time, for $b=-1, c=2, K=32, L=12, M=4, N_{x}=256, N_{y}=32, N_{z}=16, \Delta t=5 \times 10^{-4}$. Here, $\lambda_{z}=0.01,0.2,0.3,50$ and $\varepsilon=10^{-2}$.

We propose now to extend our experiments to the case of very large values of the wavelength in order to inspect numerically if the following aspect, (theoretically) established in the context of lump-solitons (see [6]), occurs in the presence of Zaitsev waves with our considerations. Of course following [6], for a similar perturbation applied to a lump-soliton, when $\lambda_{z}$ tends to infinity, the formal limit of the perturbed lump-soliton provides a new lump-soliton, independent of the second transverse variable. Figure 50 presents the results obtained by considering then $\lambda_{z}=10^{8}, 10^{9}$.
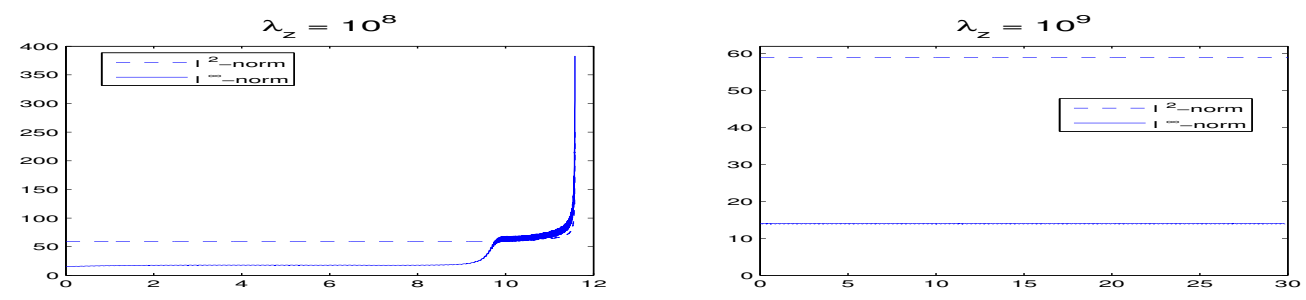

Figure 50: Evolutions of the $l^{2}$-norm (--) and the $l^{\infty}$-norm (-) with respect to time, for $b=-1, c=2, K=32, L=12, M=4, N_{x}=256, N_{y}=32, N_{z}=16, \Delta t=5 \times 10^{-4}$. Here, $\lambda_{z}=10^{8}, 10^{9}$ and $\varepsilon=10^{-2}$.

We thus observe here a phenomenon similar to the one noted in the context of lump-solitons. In fact, there exists another critical transverse wavelength $\lambda_{c}^{2}$ such that if $\lambda_{z}>\lambda_{c}^{2}$, the perturbed Zaitsev wave no longer blows up.

The above observations are not influenced by the choice of a smaller value of $\varepsilon$. Of course, 
as Figure 51 shows, the evolutions of the $l^{2}$ and $l^{\infty}$-norms, obtained by considering for instance $\varepsilon=5 \times 10^{-3}$ in simulations, do not qualitatively differ from those presented in Figure 48. Similar results to those of Figures 49 and 50 derive also from simulations when $c=2$ and $\varepsilon=5 \times 10^{-3}$.
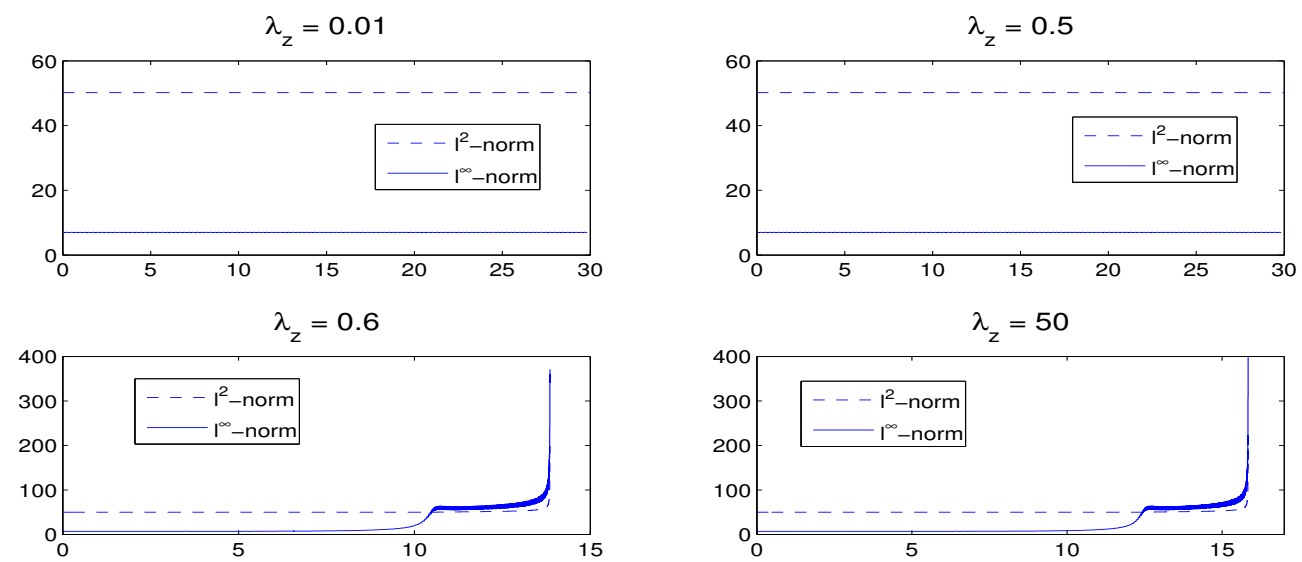

Figure 51: Evolutions of the $l^{2}$-norm (--) and the $l^{\infty}$-norm (-) with respect to time for $b=-1, c=1, K=32, L=12, M=4, N_{x}=256, N_{y}=32, N_{z}=16, \Delta t=5 \times 10^{-4}$. Here, $\lambda_{z}=0.01,0.5,0.6,50$ and $\varepsilon=5 \times 10^{-3}$.

In the last context where $b=1$, the perturbed Zaitsev wave seems to never blow up. The results of Figures 52 and 53 are obtained from experiments performed in this context by setting $c=2$; similar results derive also from simulations when we consider $c=1$.
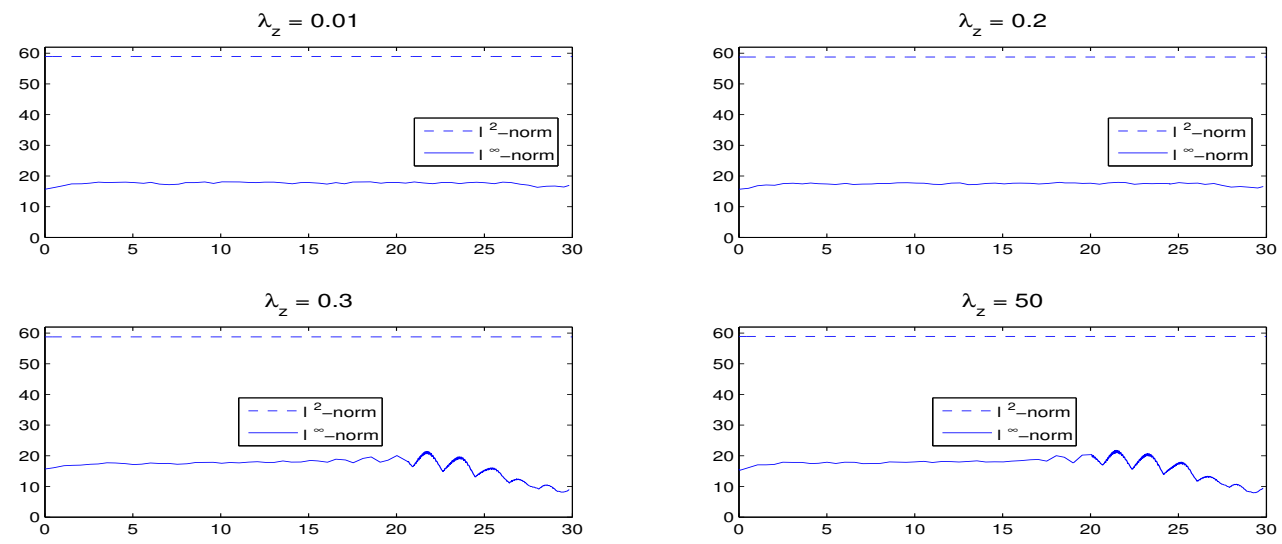

Figure 52: Evolutions of the $l^{2}$-norm (--) and the $l^{\infty}$-norm (-) with respect to time, for $b=1, c=2, K=32, L=12, M=4, N_{x}=256, N_{y}=32, N_{z}=16, \Delta t=5 \times 10^{-4}$. Here, $\lambda_{z}=0.01,0.2,0.3,50$ and $\varepsilon=10^{-2}$. 

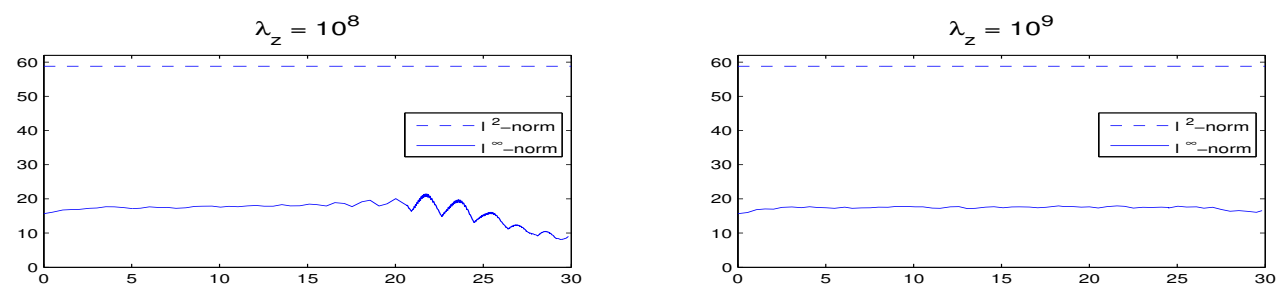

Figure 53: Evolutions of the $l^{2}$-norm (--) and the $l^{\infty}$-norm (-) with respect to time, for $b=1, c=2, K=32, L=12, M=4, N_{x}=256, N_{y}=32, N_{z}=16, \Delta t=5 \times 10^{-4}$. Here, $\lambda_{z}=10^{8}, 10^{9}$ and $\varepsilon=10^{-2}$.

The dispersive effect prevails over the blow-up, and it seems, when $\lambda_{c}^{1}<\lambda_{z}<\lambda_{c}^{2}$, that another form of stability appears.

\subsection{Periodicity in time of the crest}

In the context where $b=-1$, a periodicity in time of the perturbed Zaitsev wave's oscillations is observed when the wavelength $\lambda_{z}$ is such that $c \lambda_{z}<0.6$. Indeed, in a reference frame moving at the velocity $c$, the amplitude of the top wave, $\sup _{x, y} u(x, y, z, 0)$, is given by

$$
A_{c}(z)=6\left(c\left(1+\varepsilon \cos \left(\frac{2 \pi z}{\lambda_{z}}\right)\right) \pm \sqrt{c^{2}\left(1+\varepsilon \cos \left(\frac{2 \pi z}{\lambda_{z}}\right)\right)^{2}-4 \delta^{2}}\right)
$$

and represents the oscillations of the perturbed Zaitsev wave's crest around the value $6\left(c \pm \sqrt{c^{2}-4 \delta^{2}}\right)$.

Let us consider here $c=1,2$, and fix $\varepsilon=10^{-2}$ in our experiments, where the values of $K, L, M, N_{x}, N_{y}, N_{z}$ and $\Delta t$ are the same as previously. Figure 54 presents the evolution of $\sup _{x, y} u(x, y, 0, t)$, where $u$ is the discrete solution obtained respectively with $c=1, \lambda_{z}=0.2$, 0.5 , and $c=2, \lambda_{z}=0.2$. 

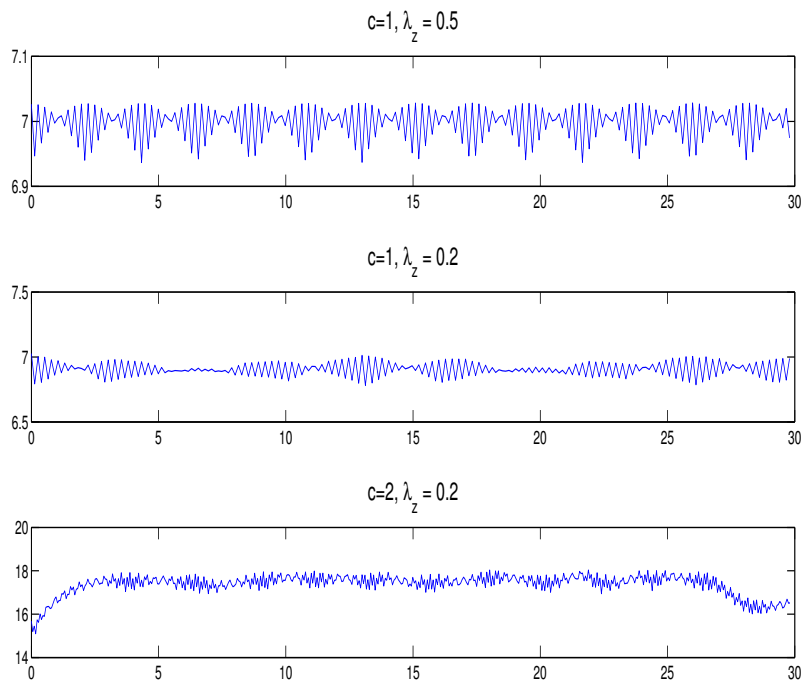

Figure 54: Evolution of $\sup _{x, y} u(x, y, 0, t)$ with respect to time, for $b=-1, c=1,2, K=32, L=$ $12, M=4, N_{x}=256, N_{y}=32, N_{z}=16, \Delta t=5 \times 10^{-4}$. Here, $\lambda_{y}=\lambda_{z}=0.5,0.2$ and $\varepsilon=10^{-2}$.

For $c$ fixed, the period increases when $\lambda_{z}$ decreases. On the other hand, for $\lambda_{z}$ fixed, the period increases when $c$ decreases.

At fixed instants $(T=2.043,15.841)$, Figure 55 shows the values of $\sup _{x, y} u(x, y, z, T)$, where $u$ is the obtained discrete solution for $\lambda_{z}=0.2, c=2$.
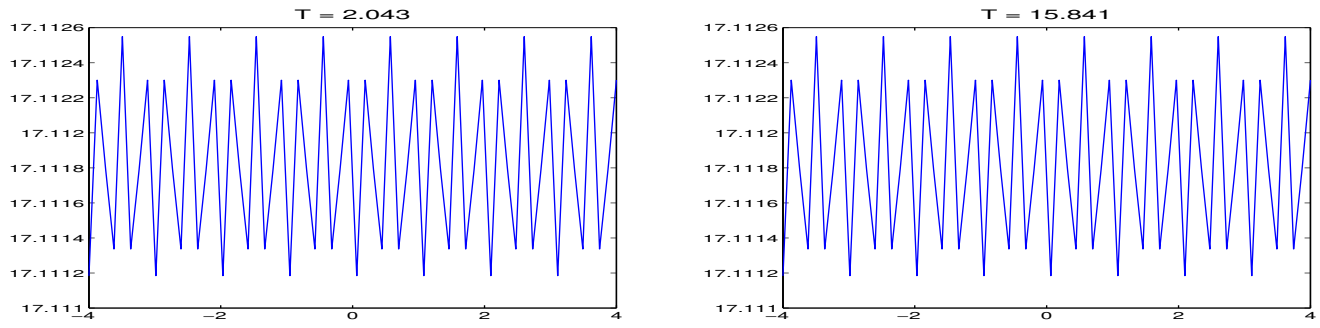

Figure 55: Representations of $\sup _{x, y} u(x, y, z, T)$ with respect to $z$, for $T=2.043,15.841$, with $b=-1, c=2, K=32, L=12, M=4, N_{x}=256, N_{y}=32, N_{z}=16, \Delta t=5 \times 10^{-4}$. Here, $\lambda_{z}=0.2$ and $\varepsilon=10^{-2}$.

The results obtained from experiments performed in the last context, namely with $b=1$, are represented in the last figure. It appears that the periodicity is lost at the instant $T \simeq 15$. We notice, in comparison with Figure 52, that from this instant, the solution has a tendency to blow up. 

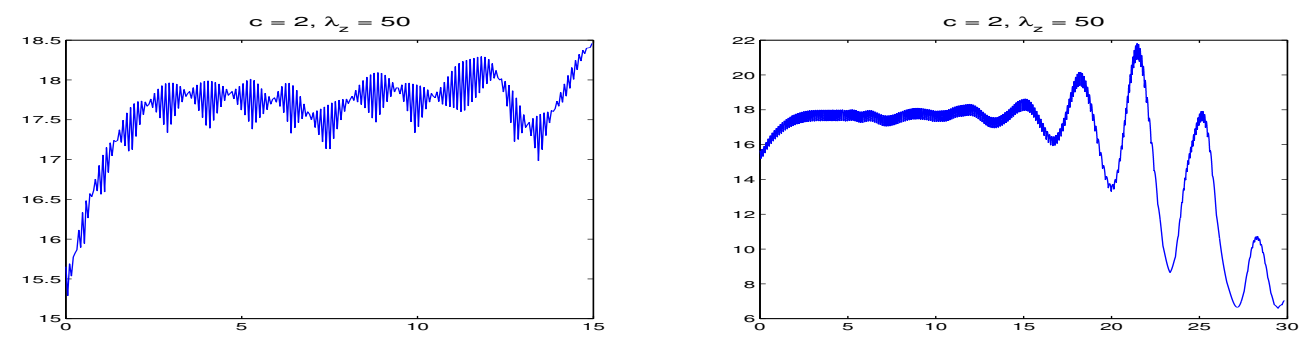

Figure 56: Evolution of $\sup _{x, y} u(x, y, 0, t)$ with respect to time, for $b=1, c=2, K=32, L=$ $12, M=4, N_{x}=256, N_{y}=32, N_{z}=16, \Delta t=5 \times 10^{-4}$. Here, $\lambda_{z}=50$ and $\varepsilon=10^{-2}$.

Let us summarize now the numerical observations of this section. It follows that the wavelength $\lambda_{z}$ characterizes the instability of the Zaitsev wave by transverse perturbations in the $z$-direction. More precisely, when $b=-1$, there exist two critical transverse wavelengths $0<\lambda_{c}^{1} \ll \lambda_{c}^{2}$, depending only on $c$, with $\lambda_{c}^{1} \simeq \frac{0.6}{c}$, such that the corresponding discrete solution blows up in finite time if $\lambda_{c}^{1}<\lambda_{z}<\lambda_{c}^{2}$. When $b=1$, the solution of (2), with the perturbed Zaitsev wave as initial datum, does not blow up.

We can then formulate the following conjecture.

\section{Conjecture 5.1}

For $\varepsilon>0$ sufficiently small, let $u_{0}$ be the perturbed Zaitsev wave of velocity $c(z):=c(1+$ $\left.\varepsilon \cos \left(\frac{2 \pi z}{\lambda_{z}}\right)\right)$, where $\lambda_{z}>0$. Let $p=1$, and $a=b=-1$. There exist two critical transverse wavelengths $0<\lambda_{c}^{1} \ll \lambda_{c}^{2}$, depending only on $c$, such that the solution of the $3 D$-KP equation (2), with $u_{0}$ as initial datum, blows up in finite time (in particular the Zaitsev wave is unstable under the flow of (2)) if $\lambda_{c}^{1}<\lambda_{z}<\lambda_{c}^{2}$.

\section{General conclusion}

The numerical experiments performed in this paper have allowed us to study the time behavior of solutions of (2), $u_{t}+u^{p} u_{x}+u_{x x x}+a \partial_{x}^{-1} u_{y y}+b \partial_{x}^{-1} u_{z z}=0$, with respect to the parameters $a$, $b, a b= \pm 1, p \geq 1$, and various initial data. Our results show that these solutions could disperse to zero, or, on the contrary, blow up in finite time (prevalence of the non-linearity), or again, approach an equilibrium state (solitonic behavior).

We have first followed the evolution of solutions of (2) for localized initial data, defined as $u_{0}(x, y, z)=\alpha\left(1-2 s x^{2}\right) \mathrm{e}^{-s\left(x^{2}+y^{2}+z^{2}\right)}$, with $\alpha, s \in \mathbb{R}_{+}^{\star}$. In the context where $a=b=-1$, the solution blows up from $p=2$. On the other hand, the effect of two positive or "opposite" transverse directions is much more regularizing, in the sense that the solution blows up from $p=3$ when $a=b=1$ or $a b=-1$. A solitonic behavior of the solution is observed when 
$a=b=-1$, for a large amplitude of the initial datum. In all other experiments, the solution disperses.

We have also studied transverse instabilities. The considered initial data are then perturbed line-solitons, defined as $u_{0}(x, y, z)=\left(\frac{(p+1)(p+2)}{2} c(y, z)\right)^{1 / p} \operatorname{sech}^{2 / p}\left(\frac{p \sqrt{c(y, z)}}{2} x\right)$, where $c(y, z):=$ $c\left(1+\varepsilon \cos \left(\frac{2 \pi y}{\lambda_{y}}+\frac{2 \pi z}{\lambda_{z}}\right)\right)$, with $c>0$ and $\varepsilon>0$ a fixed small value. The numerical experiments, in each of the contexts where $p=1,2$ with $a=b=-1$ or $p=2$ with $a b=-1$, suggest the existence of two critical wavelengths $0<\lambda_{c}^{1} \ll \lambda_{c}^{2}$, depending only on $c$, such that the solution of (2) blows up in finite time if $\lambda_{c}^{1}<\lambda_{y}<\lambda_{c}^{2}$ or $\lambda_{c}^{1}<\lambda_{z}<\lambda_{c}^{2}$. Blow-up is also observed when $p=1$ with $a b=-1$, for particular wavelengths $\lambda_{y}, \lambda_{z}$. In the presence of very small or very large wavelengths, the solution does not blow up. Its evolution, in the context of very small wavelengths, consists of periodical oscillations.

Finally, we have explored a new type of transverse instability. Now, the initial data are perturbed Zaitsev traveling waves, defined as $u_{0}(x, y, z)=12 \alpha(z)^{2} \frac{1-\beta(z) \cosh (\alpha(z) x) \cos (\delta y)}{(\cosh (\alpha(z) x)-\beta(z) \cos (\delta y))^{2}}$, where $\beta(z)=\sqrt{\frac{\delta^{2}-3 \alpha(z)^{4}}{\delta^{2}}}, 3 \alpha(z)^{4}<\delta^{2}, c(z) \alpha(z)^{2}=\alpha(z)^{4}+\delta^{2}$, and $c(z):=c\left(1+\varepsilon \cos \left(\frac{2 \pi z}{\lambda_{z}}\right)\right)$, with $\delta>0$ as $c>0$ fixed, and $\varepsilon>0$ a fixed small value. Here again, for $p=1$ with $a=b=-1$, there exist two critical wavelengths $0<\lambda_{c}^{1} \ll \lambda_{c}^{2}$, depending only on $c$, such that the solution of (2) blows up if $\lambda_{c}^{1}<\lambda_{z}<\lambda_{c}^{2}$. Moreover, when the wavelength does not satisfy the "instability condition", the evolution of the solution consists of periodical oscillations.

\section{References}

[1] J.C. Alexander, R.L. Pego \& R.L. Sachs, "On the transverse instability of solitary waves in the KadomtsevPetviashvili equation", Phys. Lett. A, A 226, (1997), 187-192.

[2] T.B. Benjamin, "The stability of solitary waves", Proc. Roy. Soc. Ser. A, 328, (1972), 153-183.

[3] J. Bourgain, "On the Cauchy problem for the Kadomtsev-Petviashvili equations", Geometric and Functional Anal., 3,4, (1993), 315-341.

[4] A. De Bouard \& J.-C. Saut, "Solitary waves of generalized Kadomtsev-Petviashvili equations", Annales Institut Henri Poincaré, Analyse Non Linéaire, 4 (1997), 211-236.

[5] A. De Bouard \& J.-C. Saut, "Symmetries and decay of the generalized Kadomtsev-Petviashvili solitary waves", SIAM J. Math. Anal., 28,5, (1997), 1064-1085.

[6] A. De Bouard \& J.-C. Saut, "Remarks on the stability of generalized KP solitary waves", Mathematical problems in the theory of water waves (Luminy, 1995), 200, (1996), 75-84.

[7] A.V. Faminskii, "The Cauchy problem for the generalized Kadomtsev-Petviashvili equation", (Russian) Sibirsk. Mat. Zh., 33, (1992), 160-172, 221; translation in Siberian Math. J., 33, (1992), 133-143.

[8] A.S. Fokas \& L.Y. Sung, "On the solvability of the N-wave, Davey-Stewartson and Kadomtsev-Petviashvili equations", Inverse Problems, 8, (1992), 673-708.

[9] F. Hamidouche, Simulations numériques des équations de Kadomtsev-Petviashvili, Ph. D. Thesis, Université Paris-Sud, 2001. 
[10] P. Isaza, J. Mejia \& V. Stallhohm, "Local solution for the Kadomtsev-Petviashvili equation in $\mathbb{R}^{2} "$, J. Math. Anal. Appl., 196, (1995), 566-587.

[11] B.B. Kadomtsev \& V.I. Petviashvili, "On the stability of solitary waves in weakly dispersing media", Soviet Physics Doklady, 15,6, (1970), 539-541.

[12] E.A. Kuznetsov \& S.L. Musher, "Effect of collapse of sound waves on the structure of collisionless shock waves in a magnetized plasma", Soviet Phys. JETP, 64,5 (1986), 947-955.

[13] Y. Liu, "Strong instability of solitary-wave solutions to a Kadomtsev-Petviashvili equation in three dimensions", J. Differential Equations, 180, (2002), 153-170.

[14] L. Molinet, J.-C. Saut \& N. Tzvetkov, "Global well-posedness for the KP-I equation on the background of a non-localized solution", Commun. Math. Phys., 272, (2007), 775-810.

[15] L. Molinet, J.-C. Saut \& N. Tzvetkov, "Remarks on the mass constraint for KP-type equations", SIAM J. Math. Anal., 39, (2007), 627-641.

[16] A. Quarteroni, R. Sacco \& F. Saleri, Numerical mathematics, Springer-Verlag, New York, 2000.

[17] F. Rousset \& N. Tzvetkov, "Transverse nonlinear instability for two-dimensional dispersive models", Annales Institut Henri Poincaré, Analyse Non Linéaire, to appear.

[18] J.-C. Saut, "Remarks on the generalized Kadomtsev-Petviashvili equations", Indiana Math. J., 42,3, (1993), 1011-1026.

[19] J.-C. Saut, "Recent results on the generalized Kadomtsev-Petviashvili equations", Acta Appl. Math., 39, (1995), 477-487.

[20] M. Tajeri \& Y. Murakami, "The periodic soliton resonance: solutions of the Kadomtsev-Petviashvili equation with positive dispersion", Phys. Lett. A, 143, (1990), 217-220.

[21] M. Tom, "On a generalized Kadomtsev-Petviashvili equation", Mathematical Problems in the Theory of Water Waves, AMS Contemporary Mathematics, 200, (1996), 193-210.

[22] S. Ukai, "Local solutions of the Kadomtsev-Petviashvili equation", J. Fac. Sci. Univ. Tokyo, Sect. IA Math., 36, (1989), 193-209.

[23] X.P. Wang, M.J. Ablowitz \& H. Segur, "Wave collapse and instability of solitary waves of generalized Kadomtsev-Petviashvili equation", Physica D, 78, (1994), 241-265.

[24] A.A. Zaitsev, "Formation of stationary waves by superposition of solitons", Sov. Phys. Dokl., 28(9), (1983), 720-722.

[25] X. Zhou, "Inverse scattering transform for the time dependent Schrödinger equation with applications to the KPI equation", Comm. Math. Phys., 128, (1990), 551-564. 